\title{
Analytical study of third-mode lateral thermal buckling for unburied subsea pipelines with
}

\author{
sleeper \\ Zhenkui Wang ${ }^{\mathrm{a}}$, G.H.M. van der Heijden ${ }^{\mathrm{b}, *}$, Yougang Tang ${ }^{\mathrm{a}}$ \\ a State Key Laboratory of Hydraulic Engineering Simulation and Safety, Tianjin University, Tianjin 300072, China \\ ${ }^{\mathrm{b}}$ Department of Civil, Environmental and Geomatic Engineering, University College London, London WC1E 6BT, UK \\ Corresponding author: G.H.M. van der Heijden, g.heijden@ucl.ac.uk
}

\begin{abstract}
Unburied subsea pipelines operating under high-temperature and high-pressure conditions tend to relieve their axial compressive force by forming lateral buckles. In order to manage lateral buckling, a sleeper is often employed as a buckleinitiation technique to ensure pipeline integrity. In this study, analytical solutions of third-mode lateral buckling for unburied subsea pipelines with sleeper are derived. The analytical solution is compared with experimental data in the literature and shows good agreement. The stability of the buckled pipeline is investigated by means of an energy analysis and it is found that third-mode lateral buckling has lower energy than first-mode buckling, which means that third-mode buckling is more likely to happen in practice. The influence of sleeper height and sleeper friction on lateral post-buckling behaviour is illustrated and analysed, with particular attention paid to the minimum critical temperature difference, lateral displacement amplitude and maximum stress. Our results show that increasing the height of the sleeper or decreasing the friction between pipeline and sleeper can both be used to decrease the minimum critical temperature difference, but their influence on the maximum stress is opposite.
\end{abstract}

Keywords: Subsea pipeline; Lateral buckling; Sleeper; Beam-column; Buckle initiation method.

\section{Nomenclature}

$P_{0}$ is the axial compressive force induced by high temperature and high pressure in sections of the pipeline where no axial expansion occurs,

$P$ is the axial compressive force in the span region,

$E$ is the elastic modulus,

$I$ is the moment of inertia of the cross-section,

$f_{A}$ is the axial soil resistance per unit length,

$l_{s}$ is the half-length of the feed-in zone,

$v$ is the vertical deflection,

$q$ is the submerged weight per unit length of the pipeline,

$l_{1}$ is half the span length,

$F$ is the shear force at the contact point between sleeper and pipeline,

$F_{s t}$ is the supporting force from the sleeper,

$v_{\text {om }}$ is the sleeper height,

$F_{t}$ is the point contact force between the pipeline and the seabed at the touchdown point,

$\bar{P}(x)$ is the axial compressive force distribution,

$\mu_{A}$ is the axial friction coefficient between pipeline and seabed,

$f_{A t}$ is the axial friction force induced by the contact vertical force $F_{t}$,

$w_{1}, w_{2}$ and $w_{3}$ are lateral deflections,

$A_{1}-A_{4}, B_{1}-B_{4}, C_{1}-C_{4}$ and $D_{1}-D_{4}$ are constant coefficients,

$f$ is the lateral friction force,

$\mu_{L}$ is the lateral friction coefficient,

$l_{2}$ is the half-length of the primary lobe, 
$l_{3}$ is the half-length of the buckled region,

$\lambda$ is the equivalent axial compressive force,

$f_{\text {ow }}$ is the lateral shear force induced by the lateral friction force between pipeline and sleeper,

$\mu_{s}$ is the friction coefficient between pipeline and sleeper,

$x$ is the distance measured along the $X$ axis,

$f_{t}$ is the lateral concentrated friction force at the touchdown point,

$f_{2}$ is the point force at $x=l_{3}$,

$T_{0}$ is the temperature difference between the fluid flowing inside the pipe and the environment,

$u_{1}$ is the length of axial expansion within the pipeline section $0<x<l_{s}$ due to high pressure and high temperature,

$u_{2}$ is the geometric shortening, which allows for the additional length introduced by the lateral displacement,

$A$ is the cross-sectional area of the pipeline,

$D$ is the external diameter of the pipe,

$\Delta \bar{P}(x)$ is the amount of decrease of axial compressive force along the pipeline after the pipeline buckles,

$\alpha$ is the coefficient of linear thermal expansion,

$u$ is the axial deformation of the pipeline,

$w_{m}$ is the maximum lateral displacement along the pipeline,

$M$ is the bending moment along the buckled pipeline,

$M_{m}$ is the maximum bending moment,

$\sigma_{M}$ is the bending stress along the buckled pipeline induced by the bending moment,

$\sigma_{M m}$ is the stress induced by the maximum bending moment,

$\sigma_{P}$ is the axial compressive stress,

$\sigma_{m}$ is the maximum stress along the pipeline induced by the axial compressive force and the maximum bending moment,

$P_{a}$ is the axial compressive force at the virtual anchors between two buckles,

$l_{a}$ is the maximum axial feed-in length,

$T_{m}$ is the minimum critical temperature difference,

$V$ is the total potential energy relating to the buckled pipeline,

$V_{i}$ is the total potential energy of the straight pipeline, namely before buckling,

$V_{1}$ is the bending strain energy,

$V_{2}$ is the energy loss due to lateral soil resistance,

$V_{3}$ is the energy loss due to axial soil resistance,

$V_{4}$ is the axial compressive strain energy,

\section{Introduction}

Subsea pipelines are increasingly being required to operate under high-temperature conditions to ease the flow and prevent solidification of the wax fraction in deep water. Due to the constraint of seabed foundation, the excessive axial compressive force will be accumulated, which may lead to lateral buckling for unburied subsea pipelines. Such uncontrolled lateral buckling may lead to fracture, fatigue or local buckling [1]. Therefore some engineering measures have been taken to prevent subsea pipeline buckling, such as trenching, burying and rock-dumping, or to relieve the stress with in-line expansion spools [2]. However, these methods are becoming more and more expensive as the operating temperature increases and as hydrocarbon development moves into deeper water [3].

Thus, an effective and inexpensive method is proposed for the relief of thermal induced axial compressive force, which is to accommodate thermal expansion by artificially inducing the pipeline to buckle in a controlled manner at several controlled locations, rather than to allow it to suffer an uncontrolled, large buckle at one location only. Thermal expansion can be evenly divided into a number of buckles, none of which is subject to too much feed-in from thermal expansion [4]. At these planned locations, a sufficient number of lateral buckles should be triggered at a sufficiently low axial compressive force [5, 6]. Several buckle initiation techniques, which are briefly described by Sinclair et al. [7], have recently been developed to ensure that 
regular buckles form along the pipeline. Three methods are commonly adopted to promote the reliable formation of lateral buckles and to control the buckle spacing and operating loads, which are snake-lay, vertical upset (sleeper) and local weight reduction through distributed buoyancy [8]. The advantage in the use of these engineered buckle initiation techniques is that the planned post-buckling configuration is generally more benign than uncontrolled lateral buckles. Consequently, the integrity of pipelines within the buckle will be improved.

Lateral and upheaval buckling have been studied previously by researchers within a theoretical framework by modelling the pipeline as a beam resting on a rigid seabed [9-16] or on a soft seabed [17-19]. Nonlinear localised lateral buckling of straight pipelines was investigated analytically by Zhu et al. [20] and Wang and van der Heijden [21] without making assumptions about the shape of lateral deformation. The research in [21] suggests that the deformed shape and the buckling path can be predicted accurately by using the assumption of a third-mode for a pipeline without sleeper, so this assumption is employed in this paper. Small-scale model tests were conducted in $[22,23]$ to understand the mechanism of upheaval buckling of buried pipelines. Experimental and numerical investigations were carried out to investigate the buckle interaction between propagation buckling and upheaval or lateral buckling in subsea pipelines by Karampour et al. [24, 25]. Many finite-element analyses have also been performed to investigate lateral and upheaval buckling [26-32]. All these works studied lateral or vertical buckling rather than how to control it.

In recent years, several researches about lateral buckling of unburied subsea pipelines with an initiation technique have been carried out. Simple analytical solutions were given for triggering lateral buckles by applying buoyancy to the pipeline by Peek and Yun [33], which are valid for a single-point buoyancy load, a two-point buoyancy load and a distributed buoyancy load over a specified length. The single-buoyancy method was further studied by Shi and Wang [5]. Analytical solutions were derived based on the first lateral buckling mode by Wang et al. [34] and on the third lateral buckling mode by Li et al. [35] and Wang et al. [36] for a pipeline section with a distributed buoyancy section. An analytical solution for controlled lateral buckling of unburied subsea pipelines was studied by Wang et al. with the consideration of interaction between adjacent buckles [4]. Moreover, experimental investigations were carried out by Silva-Junior et al. [37] and de Oliveira Cardoso et al. [38] to study the effect of distributed buoyancies and sleepers on lateral buckling. As to the study of lateral buckling for pipeline with sleeper, Wang et al. [39] derived an analytical solution based on the first lateral buckling mode. Bai et al. [40] studied the applications of dual sleepers as lateral buckling initiators through finite-element modelling. Wang et al. [41] also used finite-element modelling to analyse the buckling behaviour of pipelines with sleeper. Equations for buckling displacement, critical buckling force and buckling stress were proposed using genetic programming.

In order to verify the effect of a sleeper in controlling pipeline lateral buckling, a survey was conducted by Sinclair et al. [7] collecting operating data on the behaviour of nine pipelines employing sleepers as buckle initiators. Their results show that the sleeper initiation technique can induce both symmetric (mode 1 or 3 ) and asymmetric (mode 2) buckles (in the classification of Hobbs [9]). For mode 1, there is only a primary lobe in the positive direction, while for mode 3 there are, in addition, two adjoining secondary lobes in the negative direction. The actual mode is driven by the local imperfection introduced during pipe lay. Consequently, it is not possible to predict the buckling mode. Mode 1 has been studied analytically by Wang et al. [39]. The aim of this paper is to derive the analytical solution for the third lateral buckling mode and to compare results. First we validate our analytical model by comparing its predictions with the experimental data reported in [38], finding better agreement with our third-mode assumption than is obtained with a first-mode assumption. Then we analyse the stability of the lateral buckling solutions by computing the total energy of the pipeline. The energy of the third mode is found to be lower than that of the first mode for both pipelines with and without sleeper. Finally, parameter studies are carried out to study the effect of sleeper height and sleeper friction on the lateral buckling behaviour. 


\section{Analytical solution}

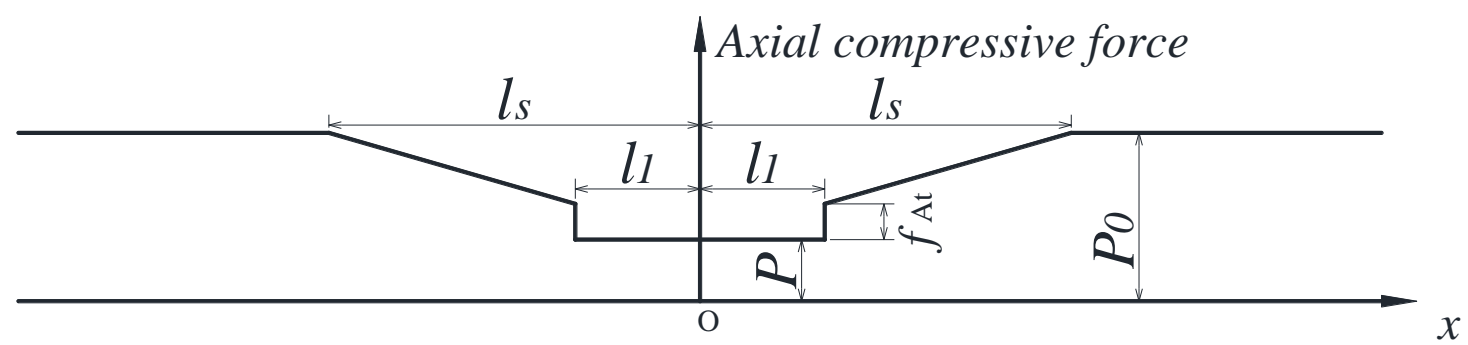

Fig. 1 Axial compressive force distribution.

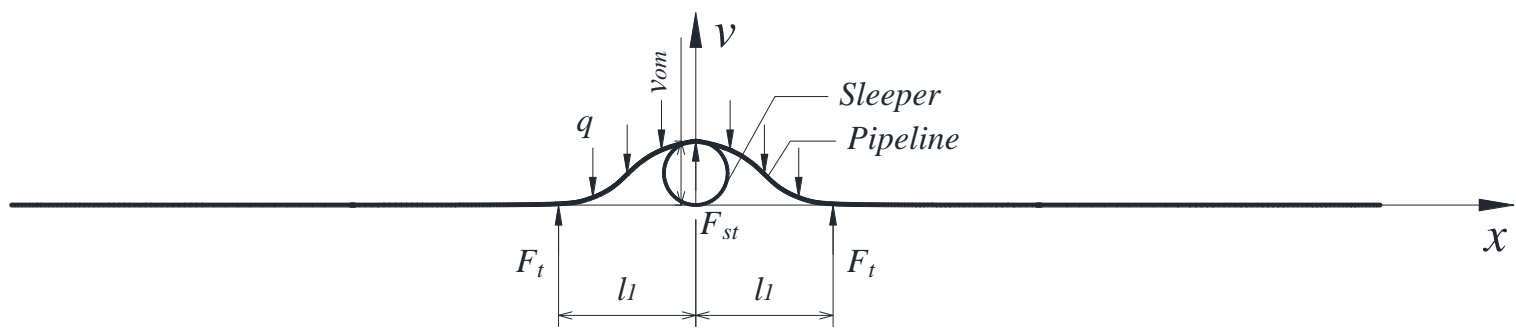

(a)

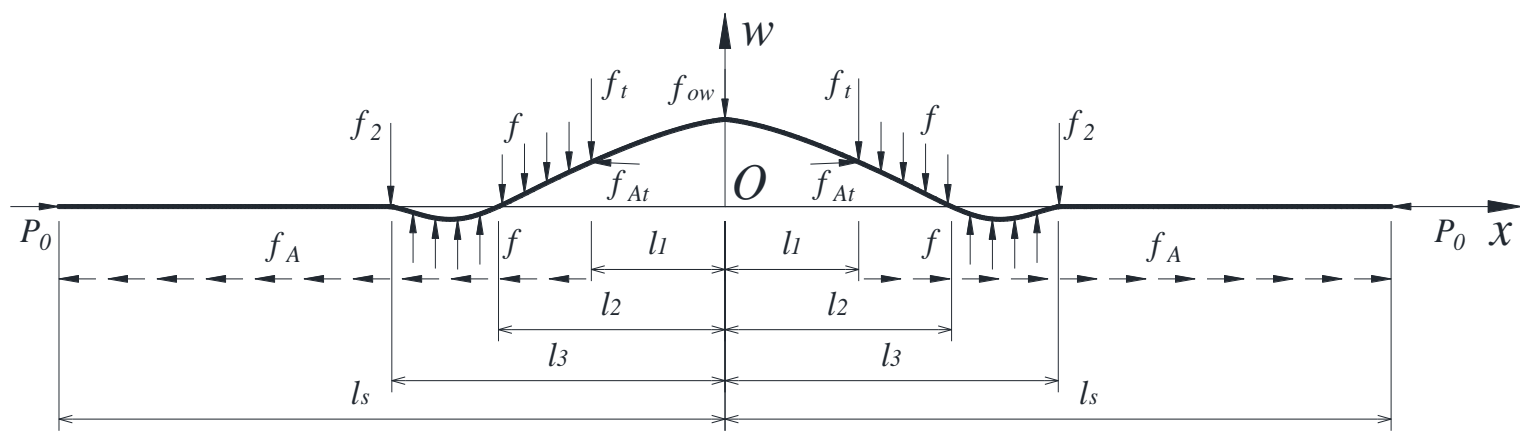

(b)

Fig. 2 Configuration and load distribution. (a) Vertical plane. (b) Horizontal plane.

In the process of thermal buckling within a pipeline section that is initially immobilised by axial friction against the seabed a small central segment of pipe will mobilise. As pipe feeds into the buckle the compressive force in the pipe drops, pulling more pipe into the buckle. If the soil resistance for axial movement is constant, say $f_{A}$, then a compressive force will build up in the pipe, increasing linearly with the distance from the touchdown point between pipeline and seabed. At some point this compressive force is sufficient to satisfy the requirement of additional length introduced by the lateral displacement. The end points of this segment are called virtual anchor points. They are the points where the axial thermal expansion drops to zero. Fig. 1 shows the feed-in region of length $2 l_{s}$ within the larger immobilised section of the pipeline together with the typical compressive force variation. $l_{s}$ is sometimes called the slip-length. $P_{0}$ is the axial compressive force at the virtual anchor points.

In practice multiple (independent) localised buckles may form in the immobilised pipe section, especially if it is long. In the following we present a theory for a single localised buckle that applies to each such buckle individually. Fig. 2 illustrates the configuration and load distribution of the third lateral buckling mode for unburied subsea pipelines with sleeper. In the 
analytical formulations of this mode presented in this section the pipeline is modelled using linear beam-column theory valid for small deflections. The vertical and lateral deflections are therefore essentially independent, coupled only by friction, and we consider each individually.

\subsection{Analytical solution in the vertical plane}

For exposed subsea pipelines the vertical resistance of the seabed is usually greater than the lateral resistance; therefore, the seabed is assumed rigid as a feasible approximation, even for soft soils. Consider a sleeper laid at the middle of the span, as shown in Fig. 2-a. The governing equation for the configuration of the pipeline with sleeper in the vertical plane is

$$
E I \frac{d^{4} v}{d x^{4}}=-q \quad 0 \leq x \leq l_{1}
$$

where $v$ is the vertical deflection, $q$ is the submerged weight per unit length of the pipeline, $E$ is the elastic modulus, $I$ is the moment of inertia of the cross-section and $l_{1}$ is half the span length. Only half of the pipeline needs to be considered owing to the symmetrical configuration and load distribution in the vertical direction.

The general solution of Eq. (1) is

$$
v=-\frac{q}{24 E I} x^{4}+D_{1} x^{3}+D_{2} x^{2}+D_{3} x+D_{4}
$$

By symmetry, the slope of $v$ at $x=0$ must be zero, while the shear force $F$ at $x=0$ comes from the supporting force $F_{s t}=2 F$ by the sleeper. In addition, the displacement, slope and bending moment at $x=l_{1}$ must be zero. So the boundary conditions at $x=0$ and $x=l_{1}$ are

$$
\left\{\begin{array}{c}
\frac{d v}{d x}(0)=0 \\
\frac{d^{3} v}{d x^{3}}(0)=\frac{F}{E I} \\
v\left(l_{1}\right)=0 \\
\frac{d v}{d x}\left(l_{1}\right)=0 \\
\frac{d^{2} v}{d x^{2}}\left(l_{1}\right)=0
\end{array}\right.
$$

Combining Eq. (2) and Eq. (3), the coefficients $D_{1}-D_{4}$ and $F$ can be obtained as

$$
D_{1}=\frac{q l_{1}}{9 E I}, D_{2}=-\frac{q l_{1}^{2}}{12 E I}, D_{3}=0, D_{4}=\frac{q l_{1}^{4}}{72 E I}, F=\frac{2}{3} q l_{1}
$$

Thus, the vertical deflection is

$$
\begin{array}{r}
v=\frac{q l_{1}^{4}}{72 E I}\left(-3 \frac{x^{4}}{l_{1}^{4}}+8 \frac{x^{3}}{l_{1}^{3}}-6 \frac{x^{2}}{l_{1}^{2}}+1\right) \\
v_{o m}=v(0)=\frac{q l_{1}^{4}}{72 E I}
\end{array}
$$

where $v_{o m}$ is the sleeper height.

From Eq. (6) we obtain

$$
l_{1}=\sqrt[4]{\frac{72 E I v_{o m}}{q}}
$$

Thus, the half-span length $l_{1}$ can be obtained through Eq. (7) when the sleeper height $v_{o m}$ is given, which will be used in the analysis of the horizontal configuration.

The point contact force between the pipeline and the seabed induced by the uplifted pipeline span at the touchdown point is

$$
F_{t}=q l_{1}-F=\frac{1}{3} q l_{1}
$$

\subsection{Analytical solution in the horizontal plane}

The pipeline consists of three zones in the horizontal plane: one span zone, in which the pipeline is uplifted by the sleeper, 
and two contacting zones, in which the pipeline contacts the seabed, as shown in Fig. 2-a. We assume that the pipeline always rests on the sleeper, i.e., that the weight of the pipe is always larger than the uplift force. Within the span zone no lateral or axial resistance is provided by the surrounding soil foundation. There is only a concentrated lateral and axial friction force at the touchdown point at $x=l_{1}$, as shown in Fig. 2-b. We assume that the value of the lateral soil resistance is constant for the pipeline lying on the seabed.

With reference to Fig. 1, the axial compressive force distribution $\bar{P}(x)$ can be expressed as

$$
\bar{P}(x)= \begin{cases}P & 0 \leq x<l_{1} \\ P+f_{A t}+f_{A}\left(x-l_{1}\right) & l_{1} \leq x<l_{S}\end{cases}
$$

Note that the axial force is constant $(P)$ in the span region because there is no distributed axial resistance acting on the pipe. For the axial soil resistance (a force per unit length) we can write

$$
f_{A}=\mu_{A} q
$$

where $\mu_{A}$ is the axial friction coefficient between pipeline and seabed. The axial friction force $f_{A t}=\mu_{A} F_{t}$ is induced by the contact vertical force $F_{t}$ at the touchdown point $x=l_{1}$.

The equation governing the horizontal deflection in the span zone is

$$
E I \frac{d^{4} w_{1}}{d x^{4}}+P \frac{d^{2} w_{1}}{d x^{2}}=0 \quad 0 \leq x \leq l_{1}
$$

where, again, by symmetry of the third buckling mode and the load distribution, only half a mode needs to be considered. For the buckled pipeline section lying on the seabed, the governing equation is

$$
\left\{\begin{array}{cl}
E I \frac{d^{4} w_{2}}{d x^{4}}+P \frac{d^{2} w_{2}}{d x^{2}}=-f & l_{1} \leq x \leq l_{2} \\
E I \frac{d^{4} w_{3}}{d x^{4}}+P \frac{d^{2} w_{3}}{d x^{2}}=f & l_{2} \leq x \leq l_{3}
\end{array}\right.
$$

where $f=\mu_{L} q$ is the lateral friction force with $\mu_{L}$ the lateral friction coefficient. Here, for the purpose of determining the deflection $w_{2}, w_{3}$, we have made the simplifying assumption that, despite Eq. (9), the axial compressive force in the entire buckled region $0 \leq x \leq l_{3}$ is constant and equal to the force $P$ at the centre of the buckle. The same approximation was made by Hobbs $[9,36,39]$. An error analysis shows that the assumption of constant axial compressive force for the calculation of lateral deflection is acceptable $[36,39]$.

Now let

$$
\lambda^{2}=\frac{P}{E I}
$$

The general solutions of Eq. (11) and Eq. (12) can then be written as

$$
\begin{gathered}
w_{1}(x)=A_{1} \cos \lambda x+A_{2} \sin \lambda x+A_{3} x+A_{4}\left(0 \leq x \leq l_{1}\right) \\
\left\{\begin{array}{l}
w_{2}(x)=B_{1} \cos \lambda x+B_{2} \sin \lambda x+B_{3} x+B_{4}-\frac{f}{2 \lambda^{2} E I} x^{2}\left(l_{1} \leq x \leq l_{2}\right) \\
w_{3}(x)=C_{1} \cos \lambda x+C_{2} \sin \lambda x+C_{3} x+C_{4}+\frac{f}{2 \lambda^{2} E I} x^{2}\left(l_{2} \leq x \leq l_{3}\right)
\end{array}\right.
\end{gathered}
$$

By symmetry, the slope of the deflection $w_{1}$ at $x=0$ must be zero, while the shear force $f_{o w}=\mu_{S} F$ at $x=0$ is induced by the friction force $2 f_{o w}$ between pipeline and sleeper. Here $\mu_{s}$ is the friction coefficient between pipeline and sleeper, also called the sleeper friction. In addition, the displacement, slope and moment at $x=l_{3}$ must be zero. So the boundary conditions at $x=0$ and $x=l_{3}$ are

$$
\left\{\begin{array}{c}
\frac{d w_{1}}{d x}(0)=0 \\
\frac{d^{3} w_{1}}{d x^{3}}(0)+\frac{f_{o w}}{E I}=0 \\
w_{3}\left(l_{3}\right)=0 \\
\frac{d w_{3}}{d x}\left(l_{3}\right)=0 \\
\frac{d^{2} w_{3}}{d x^{2}}\left(l_{3}\right)=0
\end{array}\right.
$$


The displacement, slope and bending moment must be continuous at the touchdown point $x=l_{1}$. On the other hand, there is a lateral concentrated friction force $f_{t}=\mu_{L} F_{t}$ at the touchdown point $x=l_{1}$ induced by the point contact force $F_{t}$ at $l_{1}$, so the shear force has a jump at the touchdown point $x=l_{1}$. Thus, the following matching conditions have to be satisfied at $x=l_{1}$

$$
\left\{\begin{aligned}
w_{1} & \left(l_{1}\right)=w_{2}\left(l_{1}\right) \\
\frac{d w_{1}}{d x}\left(l_{1}\right) & =\frac{d w_{2}}{d x}\left(l_{1}\right) \\
\frac{d^{2} w_{1}}{d x^{2}}\left(l_{1}\right) & =\frac{d^{2} w_{2}}{d x^{2}}\left(l_{1}\right) \\
\frac{d^{3} w_{1}}{d x^{3}}\left(l_{1}\right)= & \frac{d^{3} w_{2}}{d x^{3}}\left(l_{1}\right)+\frac{f_{t}}{E I}
\end{aligned}\right.
$$

In addition, the displacement, slope, moment and shear force must be continuous at $x=l_{2}$, while the displacement at $x=l_{2}$ must be zero. So the following matching conditions at $x=l_{2}$ are imposed:

$$
\left\{\begin{array}{c}
w_{2}\left(l_{2}\right)=w_{3}\left(l_{2}\right) \\
\frac{d w_{2}}{d x}\left(l_{2}\right)=\frac{d w_{3}}{d x}\left(l_{2}\right) \\
\frac{d^{2} w_{2}}{d x^{2}}\left(l_{2}\right)=\frac{d^{2} w_{3}}{d x^{2}}\left(l_{2}\right) \\
\frac{d^{3} w_{2}}{d x^{3}}\left(l_{2}\right)=\frac{d^{3} w_{3}}{d x^{3}}\left(l_{2}\right) \\
w_{2}\left(l_{2}\right)=0
\end{array}\right.
$$

By overall lateral force balance we find

$$
f_{2}=f\left(l_{3}-2 l_{2}+l_{1}\right)-f_{\text {ow }}-f_{t}
$$

for the point force at $x=l_{3}$, i.e., the end point of the buckled region. This $f_{2}$ is required to prevent a further lobe from forming in the horizontal plane. (Unlike in the vertical plane, the pipeline is not constrained in the horizontal plane, only resisted by friction, so in general further oscillations or lobes may form. By assuming that these do not form, i.e., that we have a third-mode laterally, we effectively consider the unbuckled part of the pipeline for $x>l_{3}$ to provide a rigid support against which the buckled part of the pipeline pushes. This requires the point force $f_{2}$ whose role is completely analogous to that of $F_{t}$ in the vertical plane.)

Axial deformation of the pipeline is governed by the equation

$$
E A \frac{d^{2} u}{d x^{2}}=f_{A} \quad\left(l_{1} \leq x \leq l_{\mathrm{s}}\right)
$$

where $A$ is the cross-sectional area of the pipe. Eq. (20) is solved subject to the slip-length boundary conditions [10]

$$
\left\{\begin{array}{c}
u\left(l_{\mathrm{s}}\right)=0 \\
\frac{d u}{d x}\left(l_{\mathrm{s}}\right)=0
\end{array}\right.
$$

giving for the axial displacement

$$
u(x)=\frac{f_{A}}{2 E A}\left(x-l_{\mathrm{s}}\right)^{2}
$$

This result will be used later when computing the total potential energy of a buckled pipe solution. Finally, by axial force balance, we have

$$
P_{0}=P+f_{A}\left(l_{s}-l_{1}\right)+f_{A t}
$$

We now use compatibility between axial and lateral deformation in the feed-in zone $0 \leq x \leq l_{s}$ to derive a relationship between the axial compressive force $P$ in the uplifted section of the pipe and the temperature difference $T_{0}$, between the fluid flowing inside the pipe and the environment, that causes the buckling. Compatibility can be expressed as

$$
u_{1}=u_{2}
$$

where $u_{1}$ is the length of axial expansion within the pipeline section $0<x<l_{s}$ due to high pressure and high temperature. $u_{2}$ is the geometric shortening, which allows for the additional length introduced by the lateral displacement. Eq. (24) simply states that, since there are virtual anchor points at distance $l_{\mathrm{s}}$ from the centre of the pipe, the extra length of pipe in the buckle 
must come from axial expansion of the mobilised segment of pipe.

We have

$$
u_{1}=\int_{0}^{l_{s}} \frac{\Delta \bar{P}(x)}{E A} d x
$$

where $\Delta \bar{P}(x)$ is the amount of decrease of axial compressive force along the pipeline after the pipeline buckles, which, from Eq. (9), is given by

$$
\Delta \bar{P}(x)= \begin{cases}P_{0}-P & 0<x<l_{1} \\ f_{A}\left(l_{s}-x\right) & l_{1}<x<l_{s}\end{cases}
$$

Thus, we find

$$
u_{1}=\frac{f_{A}\left(l_{s}-l_{1}\right)^{2}}{2 E A}+\frac{\left(P_{0}-P\right) l_{1}}{E A}
$$

Meanwhile, for $u_{2}$ we have

$$
u_{2}=\frac{1}{2} \int_{0}^{l_{1}}\left(\frac{d w_{1}}{d x}\right)^{2} d x+\frac{1}{2} \int_{l_{1}}^{l_{2}}\left(\frac{d w_{2}}{d x}\right)^{2} d x+\frac{1}{2} \int_{l_{2}}^{l_{3}}\left(\frac{d w_{3}}{d x}\right)^{2} d x
$$

Combining Eq. (24) and Eq. (27), we obtain the following equation

$$
l_{s}=\sqrt{\frac{1}{3} l_{1}^{2}+\frac{2 E A u_{2}}{f_{A}}}
$$

from which, with the use of Eq. (23) and Eq. (8), we finally obtain

$$
P_{0}=P+f_{A}\left(\sqrt{\frac{1}{3} l_{1}^{2}+\frac{2 E A u_{2}}{f_{A}}}-\frac{2}{3} l_{1}\right)
$$

Within the range of linear elastic response this compressive force $P_{0}$ can be written as

$$
P_{0}=E A \alpha T_{0}
$$

where $\alpha$ is the coefficient of linear thermal expansion. $T_{0}$ is here the total temperature difference, which is composed of the initial temperature difference and the equivalent temperature difference generated by internal pressure.

So given $T_{0}$, Eq. (31), Eq. (30) and Eq. (28) (with Eq. (14) and Eq. (15) inserted) can be solved in conjunction with Eq. (16), Eq. (17) and Eq. (18) to obtain $P, l_{2}, l_{3}$ and the coefficients $A_{1}-A_{4}, B_{1}-B_{4}$ and $C_{1}-C_{4}$, and hence the lateral deflections $w_{1}, w_{2}, w_{3}$.

We will be interested in the maximum displacement and the maximum stress along the pipeline. The stress in the pipeline consists of axial stress, $P / A$, and bending stress. The bending moment, $M$, along the buckled pipeline is given by

$$
M=E I \frac{d^{2} w}{d x^{2}}
$$

where $w$ stands for either $w_{1}, w_{2}$ or $w_{3}$, and hence the corresponding bending stress, $\sigma_{M}$, is

$$
\sigma_{M}=\frac{M D}{2 I}
$$

where $D$ is the external diameter of the pipe. The location of the maximum displacement, $w_{m}$, and maximum bending moment, $M_{m}$, can be obtained by setting $\frac{d w}{d x}=0$ and $\frac{d^{3} w}{d x^{3}}=0$. The maximum stress, $\sigma_{m}$, along the pipeline can then be computed as

$$
\sigma_{m}=\frac{P}{A}+\frac{M_{m} D}{2 I}
$$

In all the cases discussed below, these maxima occur at the centre of the pipe, i.e., at $x=0$.

\section{Analytical results}

\subsection{The values of $\lambda l_{2}$ and $\lambda l_{3}$}

The solution for the pipeline deflection $w_{1}, w_{2}$ and $w_{3}$ can be presented in semi-explicit form by using 12 of the 14 conditions in Eq. (16), Eq. (17) and Eq. (18) to express the 12 coefficients $A_{i}, B_{i}$ and $C_{i}$ in terms of $\lambda l_{2}, \lambda l_{3}$ and all the parameters of the problem (see the Appendix). The remaining two (transcendental) equations (for instance $w_{2}\left(l_{2}\right)=0$ and 
$\left.w_{3}\left(l_{3}\right)=0\right)$ can then be used to solve for the values of $\lambda l_{2}$ and $\lambda l_{3}$. By additionally solving for Eq. (30) and Eq. (31) we finally obtain the relationships between the values $\lambda l_{2}, \lambda l_{3}$ and the temperature difference $T_{0}$. For pipelines without sleeper $\left(v_{o m}=0\right)$ the values of $\lambda l_{2}$ and $\lambda l_{3}$ are 2.918 and 7.551, respectively, for all values of $T_{0}$ (see the solid lines in Fig. 3-b and Fig. 4-b). However, for pipelines with sleeper $\left(v_{o m}>0\right)$ the values of $\lambda l_{2}$ and $\lambda l_{3}$ change with the total temperature difference $T_{0}$, sleeper height $v_{o m}$ and sleeper friction $\mu_{s}$ between pipeline and sleeper. For $\mu_{s}=0.1$, the relationships between $l_{2}, l_{3}$ and $\lambda$ at various sleeper heights are given graphically in Fig. 3-a and Fig. 4-a, while the temperature dependence is shown in Fig. 3-b and Fig. 4-b. Note that conversion from $P$, i.e. $\lambda$, to $T_{0}$ leads to a fold in the solution curve: a single temperature $T_{0}$ corresponds to two different central compressive forces $P$. The influence of sleeper friction $\mu_{s}$ on the values of $\lambda l_{2}$ and $\lambda l_{3}$ is similar to that of sleeper height $v_{o m}$ and not displayed. The non-uniqueness of solutions highlights the need for a stability analysis, which we perform by means of an energy analysis in Section 3.3.

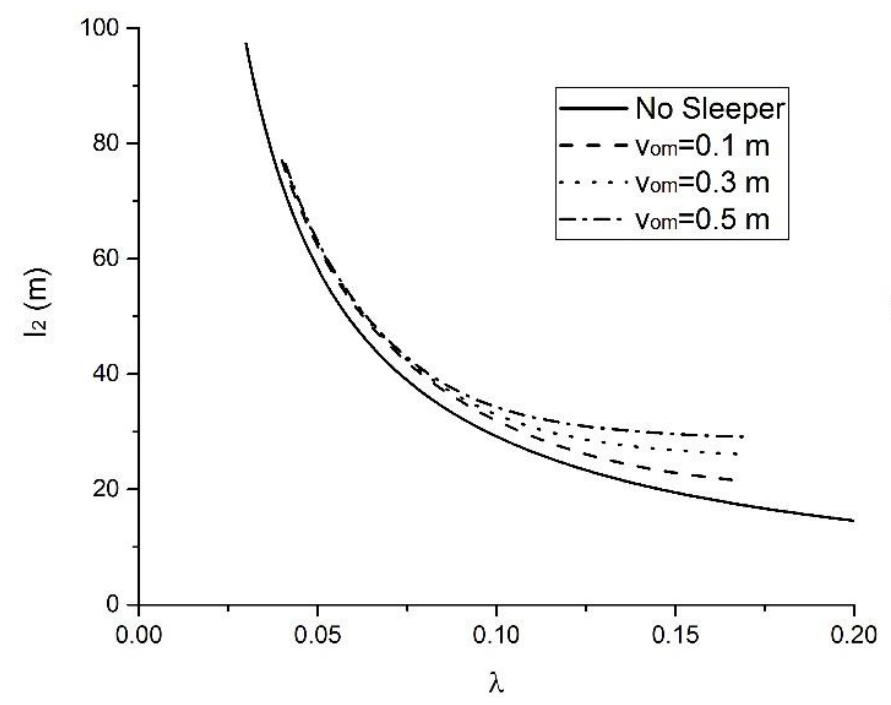

(a)

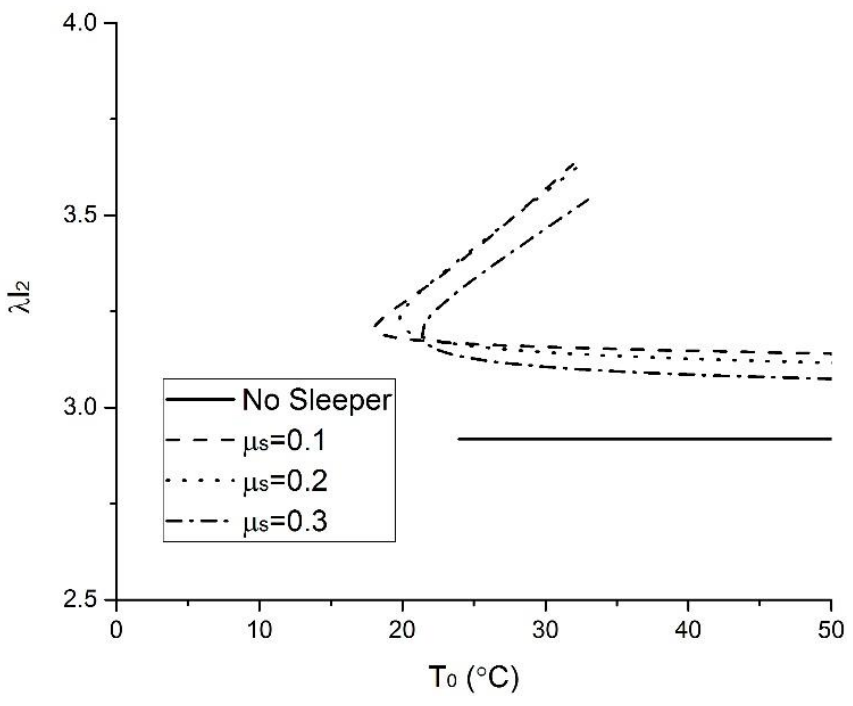

(b)

Fig. 3 The values of $\lambda l_{2}$. (a) Relationship between $l_{2}$ and $\lambda$. (b) Relationship between $\lambda l_{2}$ and $T_{0} . \mu_{s}=0.1$.

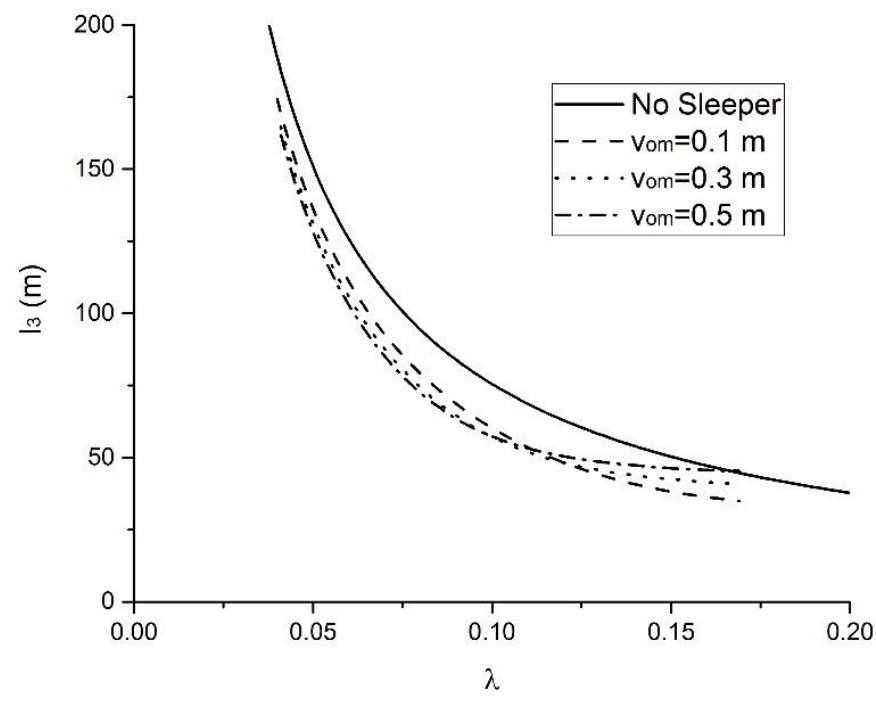

(a)

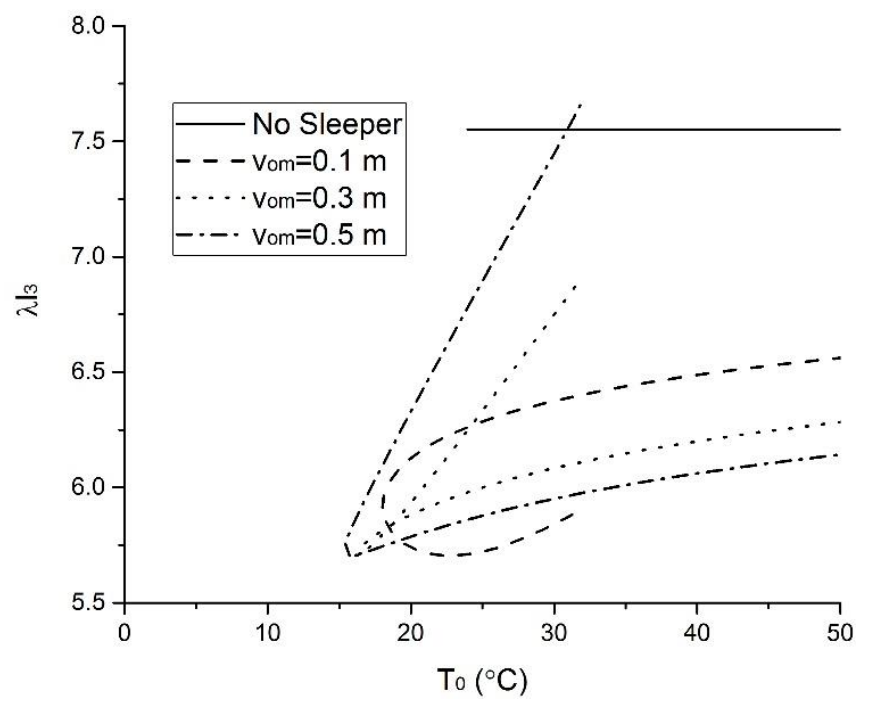

(b)

Fig. 4 The values of $\lambda l_{3}$. (a) Relationship between $l_{3}$ and $\lambda$. (b) Relationship between $\lambda l_{3}$ and $T_{0} . \mu_{s}=0.1$.

\subsection{Validation of the mathematical model}

To validate the analytical results obtained in this paper, one case is calculated by using the test parameters listed in Table 1. These parameters are the same as those used in the reduced-scale model in [38]. The total pipe length in [38] was $195 \mathrm{~m}$. Three sleepers with equal spacing of $2 l_{a}=65 \mathrm{~m}$ were installed to trigger lateral buckling. $l_{a}$ represents the maximum axial feed-in length. $P_{a}$ is the axial compressive force at the virtual anchors between two buckles. By symmetry, the axial feed-in 
displacement at the midpoint between two sleepers, namely at the virtual anchor between two buckles, is zero. So, for comparison with our model, we only consider a pipeline section over one sleeper and of length $L=65 \mathrm{~m}$. For this case, the maximum axial feed-in length $l_{a}$ is $32.5 \mathrm{~m}$. When the feed-in length $l_{s}$ is smaller than $l_{a}$, so that two adjacent buckles triggered by two adjacent sleepers are independent, all the formulae derived in this paper can be applied. When $l_{s}$ is larger than $l_{a}$, the formula for axial compressive force should be modified due to the limit of axial feed-in length. In that case, by axial force balance, we have

$$
P_{a}=P+f_{A}\left(l_{a}-l_{1}\right)+f_{A t}
$$

and the length of axial expansion within the pipeline section $0<x<l_{a}$ should be modified to

$$
u_{1}=\int_{0}^{l_{a}} \frac{\Delta \bar{P}(x)}{E A} d x
$$

where

$$
\Delta \bar{P}(x)= \begin{cases}P_{0}-P & 0<x<l_{1} \\ P_{0}-P-f_{A}\left(x-\frac{2}{3} l_{1}\right) & l_{1}<x<l_{a}\end{cases}
$$

(note that $\left.\Delta \bar{P}\left(l_{a}\right)=P_{0}-P_{a}\right)$.

Then, given $T_{0}$ and $l_{a}$, Eq. (24), Eq. (28) (with Eq. (14) and Eq. (15) inserted), Eq. (31), Eq. (36) and Eq. (37) can be solved in conjunction with Eq. (16), Eq. (17) and Eq. (18) to obtain $P, l_{2}, l_{3}$ and the coefficients $A_{1}-A_{4}, B_{1}-B_{4}$ and $C_{1}-C_{4}$, and hence the lateral deflections $w_{1}, w_{2}, w_{3}$.

The results from experimental tests and our analytical method are compared in Fig. 5 where the maximum lateral displacement $w_{m}(=w(0))$ is displayed. The present analytical results appear to agree very well with the test data. For comparison, we also include in Fig. 5 results (labelled 'infinite pipe length') obtained by assuming that always $l_{s}<l_{a}$, i.e., that the required feed-in length $l_{s}$ is always available. We see that the lateral buckling amplitude $w_{m}$ for a pipe of length 65 $\mathrm{m}$ is much smaller than that for a pipe of 'infinite length'. This explains why multiple sleepers should be installed at regular intervals to trigger pipeline buckling at multiple planned locations. The difference between the analytical and experimental responses is induced by lateral initial imperfection. The experimental curve starts from zero temperature and increases monotonically due to a large lateral imperfection, while snap-through behaviour occurs in our analytical results since no initial imperfection is considered.

Table 1. Test parameters.

\begin{tabular}{ccc}
\hline Parameter & Value & Unit \\
\hline External diameter $D$ & 15 & $\mathrm{~mm}$ \\
Wall thickness $t$ & 0.9 & $\mathrm{~mm}$ \\
Elastic modulus $E$ & 191 & $\mathrm{GPa}$ \\
Pipeline submerged weight & 3.94 & $\mathrm{~N} / \mathrm{m}$ \\
Coefficient of thermal expansion $\alpha$ & $1.75 \times 10^{-5}$ & $/{ }^{\circ} \mathrm{C}$ \\
Lateral friction coefficient $\mu_{L}$ & 0.7 & --- \\
Axial friction coefficient $\mu_{A}$ & 0.7 & --- \\
Sleeper friction $\mu_{S}$ & 0.1 & --- \\
Sleeper height $v_{o m}$ & 0.03 & $\mathrm{~m}$ \\
Pipe length $L$ & 65 & $\mathrm{~m}$ \\
\hline
\end{tabular}




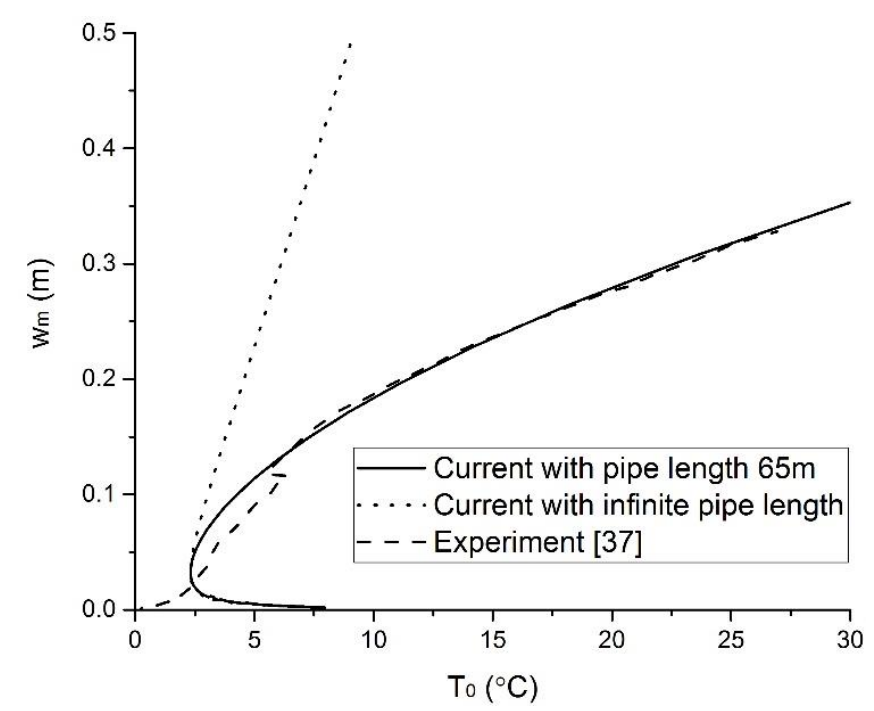

Fig. 5 Validation with test data.

\subsection{Energy analysis of a typical buckling path}

The typical relationship between lateral buckling amplitude $w_{m}$ and total temperature difference $T_{0}$ for a typical solution with sleeper is shown in Fig. 6. The significant point $m$ along the post-buckling path corresponds to the minimum critical temperature difference $T_{m} . T_{m}=18.02{ }^{\circ} \mathrm{C}$ for this case. For $T_{0}>T_{m}$ two solution branches exist, which will be referred to as $m-b$ and $m-c$, as shown in Fig. 6 . We shall compare the energies.

The total potential energy relating to the buckled pipeline (in the feed-in region $0 \leq x \leq l_{s}$ ) is given by

$$
V=V_{1}+V_{2}+V_{3}+V_{4}
$$

The bending strain energy $V_{1}$ can be expressed as

$$
V_{1}=\frac{1}{2} E I \int_{0}^{l_{1}}\left(\frac{d^{2} w_{1}}{d x^{2}}\right)^{2} d x+\frac{1}{2} E I \int_{l_{1}}^{l_{2}}\left(\frac{d^{2} w_{2}}{d x^{2}}\right)^{2} d x+\frac{1}{2} E I \int_{l_{2}}^{l_{3}}\left(\frac{d^{2} w_{3}}{d x^{2}}\right)^{2} d x
$$

The energy loss $V_{2}$ due to lateral soil resistance is

$$
V_{2}=\int_{l_{1}}^{l_{2}}\left|f w_{2}(x)\right| d x+\int_{l_{2}}^{l_{3}}\left|f w_{3}(x)\right| d x+\left|f_{o w} w_{1}(0)\right|+\left|f_{t} w_{1}\left(l_{1}\right)\right|
$$

The energy loss $V_{3}$ due to axial soil resistance is

$$
V_{3}=\int_{l_{1}}^{l_{s}}\left|f_{A} u(x)\right| d x+\left|f_{A t} u\left(l_{1}\right)\right|
$$

The axial compressive strain energy $V_{4}$ is

$$
V_{4}=\frac{1}{2 E A} \int_{0}^{l_{s}} \bar{P}(x)^{2} d x
$$

while the total potential energy of the straight pipeline, namely before buckling, is given by

$$
V_{i}=\frac{1}{2 E A} \int_{0}^{l_{s}} P_{0}^{2} d x
$$




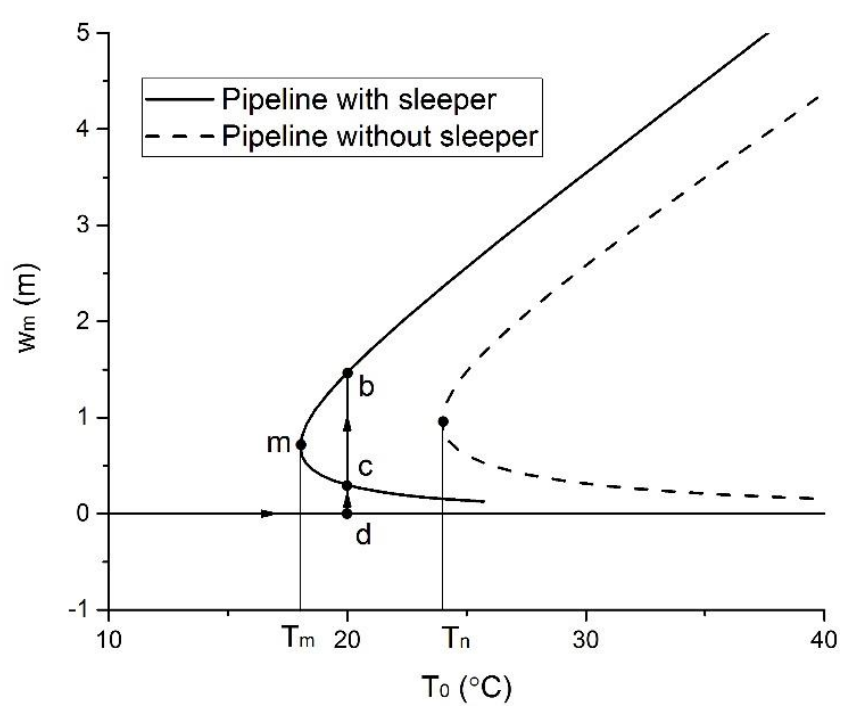

Fig. 6 Typical buckling path. $v_{o m}=0.1 \mathrm{~m} . \mu_{s}=0.1$.

When $T_{0}$ is lower than $T_{m}$ only the trivial state $\left(w_{m}=0\right)$ exists and no lateral buckling occurs. The pipeline remains in the vertical plane. However, when $T_{0}$ is larger than $T_{m}$, two lateral buckling states exist. Take $T_{0}=20^{\circ} \mathrm{C}$, for example. When $T_{0}$ reaches $20^{\circ} \mathrm{C}$, the pipeline will remain unbuckled in the absence of a disturbance or imperfection, corresponding to point $d$ in Fig. 6. However, the deformed states $b$ and $c$ are available as well and a sufficiently large disturbance may cause a jump from $d$ to one of these buckled states. From the fact that the post-buckling branch does not intersect the trivial branch we deduce that there is no critical temperature $T_{0}$ at which the pipe starts gradually to move sideways on the sleeper. Instead, the pipe will lift off the sleeper and then, at slightly higher $T_{0}$, fall sideways back onto the sleeper. Thus a jump occurs from $d$ to a point near $b$ or $c$ in Fig. 6. The total energy is calculated through Eq. (38) to determine the relative stability of the two branches.

The total energy of branches $m-b$ and $m$-c for the lateral post-buckling state are denoted by $V_{b}$ and $V_{c}$, respectively. $V_{i b}$ and $V_{i c}$ are the total potential energies of the unbuckled pipeline with sleeper of corresponding length $l_{s} . V_{b} / V_{i b}$ and $V_{c} / V_{i c}$ are illustrated in Fig. 7. We see that all the values of $V_{b} / V_{i b}$ are less than those of $V_{c} / V_{i c}$, which means that the branch $m-b$ is more stable than branch $m-c$. In addition, the value of $V_{c} / V_{i c}$ first increases slightly and then decreases with increasing temperature difference, while all the values of $V_{c} / V_{i c}$ are larger than 1 , which means that branch $m$-c is less stable than the trivial solution. The value of $V_{b} / V_{i b}$ decreases with increasing temperature difference, which means that the branch $m-b$ becomes more stable with increasing temperature difference. $V_{b} / V_{i b}=1$ when the temperature difference reaches $T_{e}=18.95^{\circ} \mathrm{C}$. For $T_{0}<T_{e}, V_{b} / V_{i b}$ is bigger than 1 , which means that the trivial solution is more stable. For $T_{0}>$ $T_{e}, V_{b} / V_{i b}$ is smaller than 1 , which means that the branch $m-b$ is more stable than the trivial state. We also note that the energy ratio for a pipeline with sleeper is smaller than that for a pipeline without sleeper, which means that the pipeline with sleeper is more stable than the pipeline without sleeper.

Fig. 6 also displays the buckling curve for the same pipeline without sleeper. We see that for $T_{0}>T_{n}$ two deformed states are available and an energy analysis shows that again the upper branch contains solutions with lower energy than the trivial state. A pipeline without sleeper, therefore, would likely jump into a buckled state under a sufficiently large disturbance when $T_{0}$ becomes larger than $T_{n}$. This buckling would be sudden, without any warning signs, and could happen at any point of a long immobilised pipeline. This potentially dangerous scenario is avoided by using a sleeper, which forces the pipeline into a deformed state at a specific point and at lower $T_{0}$ before the critical temperature $T_{n}$ is reached. We also see in Fig. 6 that the amplitude of lateral deflection is larger in the case of a sleeper (at the same temperature difference $T_{0}$ ). The effect of the sleeper will be investigated in more detail in Section 3.5. 


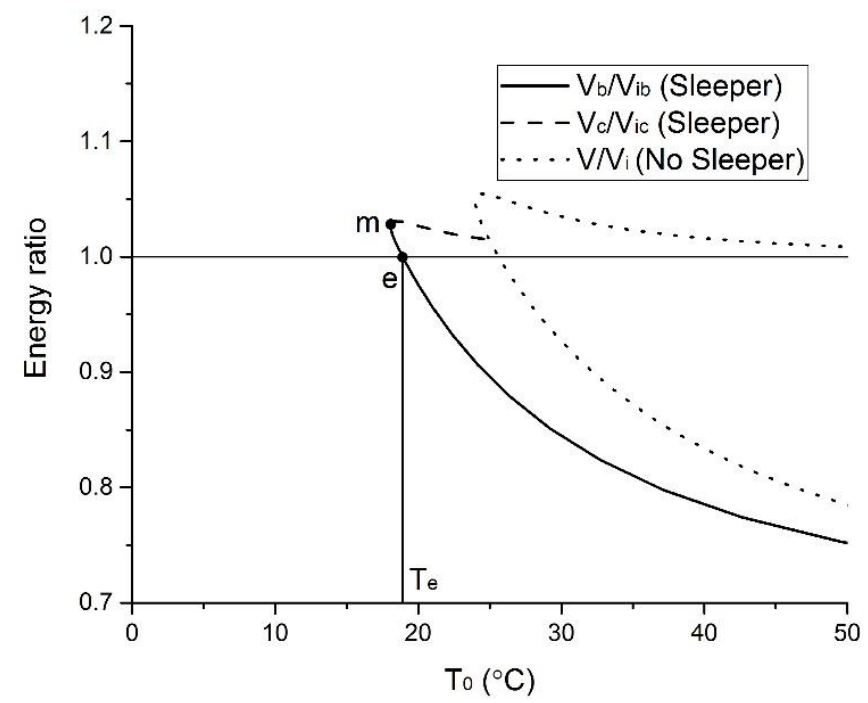

Fig. 7 Energy ratio between buckled and pre-buckling states. $v_{\text {om }}=0.1 \mathrm{~m} . \mu_{s}=0.1$.

\subsection{Comparison between mode 1 and mode 3}

In this section, first and third lateral buckling modes of subsea pipelines with and without sleeper are compared, as shown in Fig. 8. The results for the first buckling mode are obtained from [39]. The results for the third mode without sleeper are obtained by setting $v_{\text {om }}=0 \mathrm{~m}$. In Fig. 8 -a we observe that for half a buckled pipeline there is only a primary lobe in the positive direction $(w>0)$ for mode 1 ; however, a secondary lobe in the negative direction $(w<0)$ exists for mode 3 . Fig. 8 $\mathrm{b}$ shows that the minimum critical temperature difference $T_{m}$ of mode 3 is smaller than that of mode 1 for pipelines both with and without sleeper. The lateral displacement amplitude of mode 3 is also smaller than that of mode 1 at the same temperature difference. However, this difference becomes smaller when a sleeper is applied. The reason for this is that the primary lobe in the positive direction becomes larger and the secondary lobe in the negative direction becomes smaller for mode 3 when a sleeper is applied. This causes the mode-3 buckled shape of pipelines with sleeper to be close to mode 1 . In Fig. 8-c we see that the energy ratio $V / V_{i}$ of mode 3 is smaller than that of mode 1 for pipelines both with and without sleeper, which means that mode 3 is more stable than mode 1 . So mode 3 is more likely to happen than mode 1 for subsea pipelines with or without sleeper.

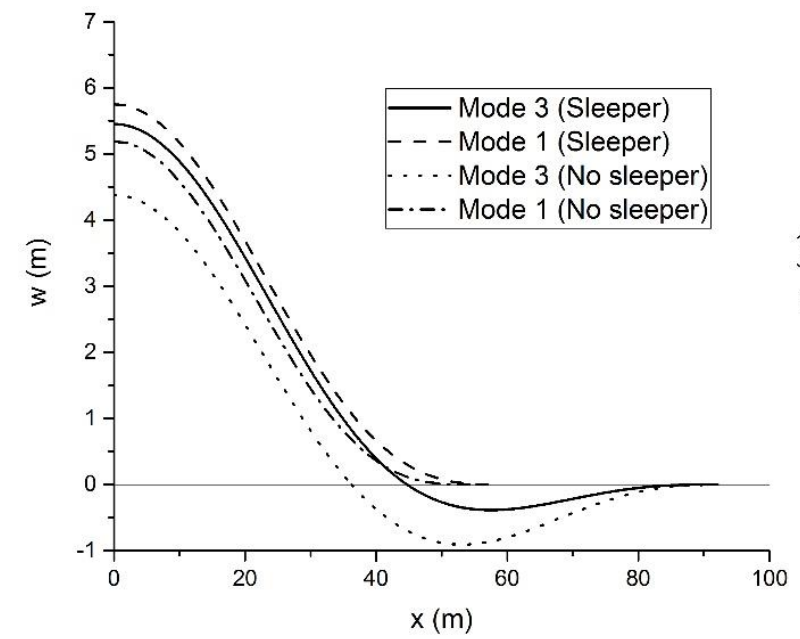

(a)

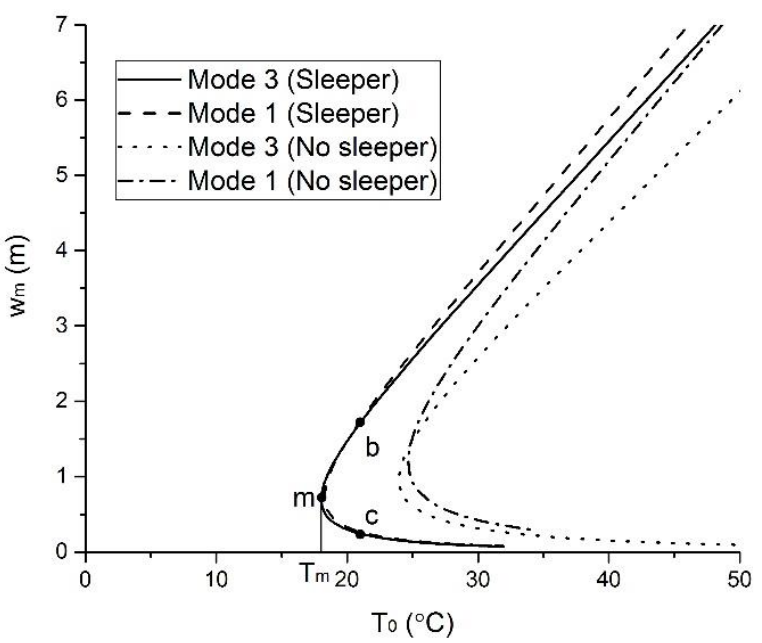

(b) 


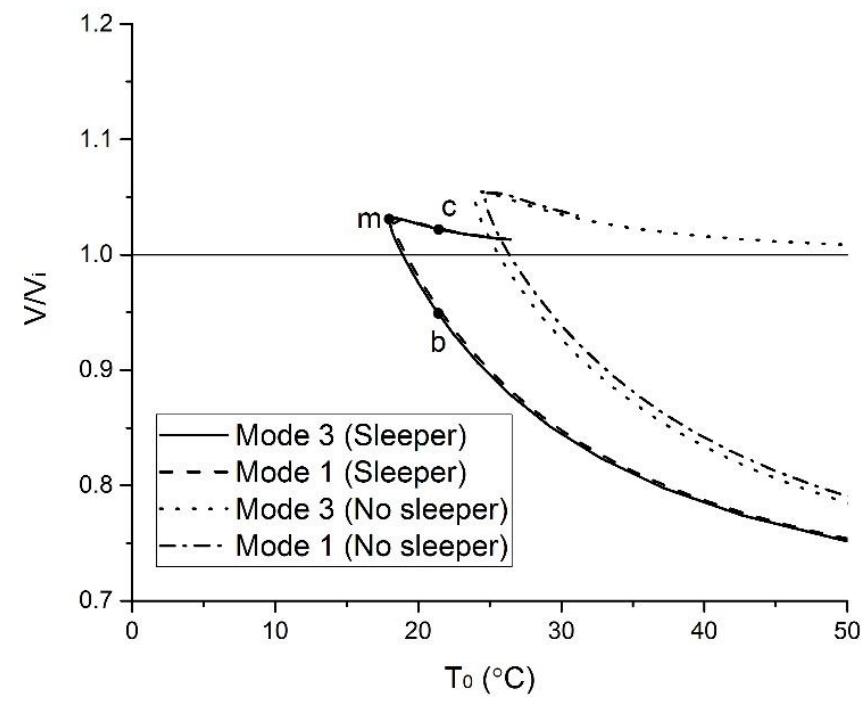

(c)

Fig. 8 Comparisons between mode 1 and mode 3. (a) Deformed shapes. (b) Buckling paths. (c) Energy ratios $V / V_{i}$. $v_{o m}=$ $0.1 \mathrm{~m} . \mu_{s}=0.1$.

\subsection{Parametric study}

Table 2. Design parameters.

\begin{tabular}{ccc}
\hline Parameter & Value & Unit \\
\hline External diameter $D$ & 323.9 & $\mathrm{~mm}$ \\
Wall thickness $t$ & 12.7 & $\mathrm{~mm}$ \\
Elastic modulus $E$ & 206 & $\mathrm{GPa}$ \\
Steel density $\rho$ & 7850 & $\mathrm{~kg} / \mathrm{m}^{3}$ \\
Coefficient of thermal expansion $\alpha$ & $1.1 \times 10^{-5}$ & $/{ }^{\circ} \mathrm{C}$ \\
Lateral friction coefficient $\mu_{L}$ & 0.5 & --- \\
Axial friction coefficient $\mu_{A}$ & 0.5 & --- \\
Sleeper friction $\mu_{S}$ & $0.1 / 0.2 / 0.3$ & --- \\
Sleeper height $v_{o m}$ & $0.1 / 0.3 / 0.5$ & $\mathrm{~m}$ \\
\hline
\end{tabular}

In this section, a typical pipeline with sleeper resting on the seabed is analysed in its third lateral buckling mode. The deformed shapes and bending stresses along the pipeline with different sleeper height $v_{o m}$ and sleeper friction $\mu_{s}$ under the same temperature are analysed and discussed first. Then the influence of sleeper height $v_{o m}$ and sleeper friction $\mu_{s}$ on typical buckling behaviour is presented and the component of maximum stress is analysed. Finally, a more detailed parametric analysis of the minimum critical temperature difference $T_{m}$, the lateral displacement amplitude $w_{m}$ and the maximum stress $\sigma_{m}$ is presented and discussed. We demonstrate this sleeper effect by employing the analytical formulation developed in Section 2 taking the parameters in Table 2 as a realistic case study. In this section, all the analysis is based on branch $\mathrm{m}-\mathrm{b}$, namely the stable branch.

\subsubsection{Buckled configuration}

The deformed shapes and the corresponding bending stresses $\sigma_{M}$ along the buckled pipeline with different sleeper height under the same operating temperature difference are presented in Fig. 9-a and Fig. 9-b, respectively. In Fig. 9-a it is seen that a localised buckled shape is formed within a limited region in the middle of the pipeline due to the axial compressive force induced by the temperature difference. This buckled shape consists of half a primary lobe in the positive direction $(w>0)$ and a secondary lobe in the negative direction $(w<0)$ for half a buckled pipeline. The maximum lateral displacement is attained at $x=0 \mathrm{~m}$ for each case. From Fig. 9-a we note that the primary lobe for the pipeline with sleeper is larger than that for the pipeline without sleeper and increases further with increasing sleeper height. However, the secondary lobe for the 
pipeline with sleeper is smaller than that for the pipeline without sleeper and decreases further with increasing sleeper height. The deformed shapes are thus closer to mode 1 for higher sleeper heights. From Fig. 9-b we see that there are three maxima of bending stress in either the positive or negative direction. The maximum bending stress is attained at $x=0 \mathrm{~m}$, so the integrity of the pipeline will be lost first at the the centre of the pipe. The bending stress at $x=0 \mathrm{~m}$ for the case $v_{o m}=$ $0.1 \mathrm{~m}$ is slightly larger than that for the pipeline without sleeper but decreases below this value with further increase of $v_{\text {om }}$, as shown in Fig. 9-b. By contrast, the sleeper height barely has an influence on the other two maxima of bending stress.

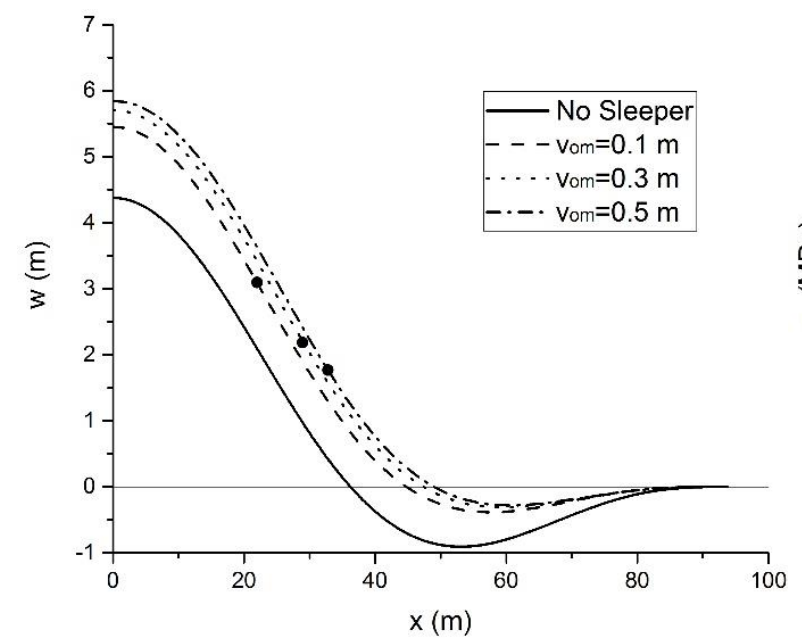

(a)

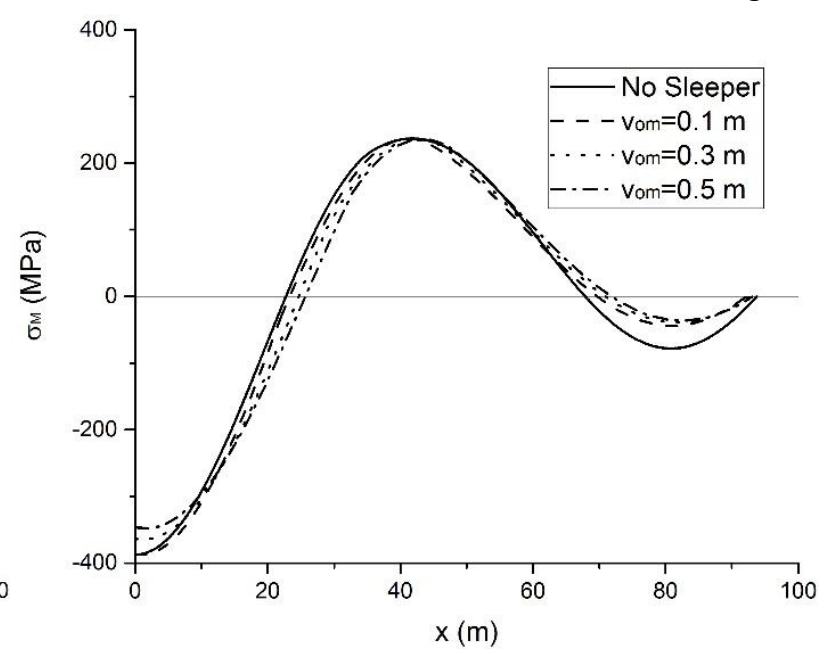

(b)

Fig. 9 Influence of sleeper height. (a) Deformed shapes. (b) Bending stress. $\mu_{s}=0.1 . T_{0}=40^{\circ} \mathrm{C}$. The dot represents the touchdown point $x=l_{1}$.

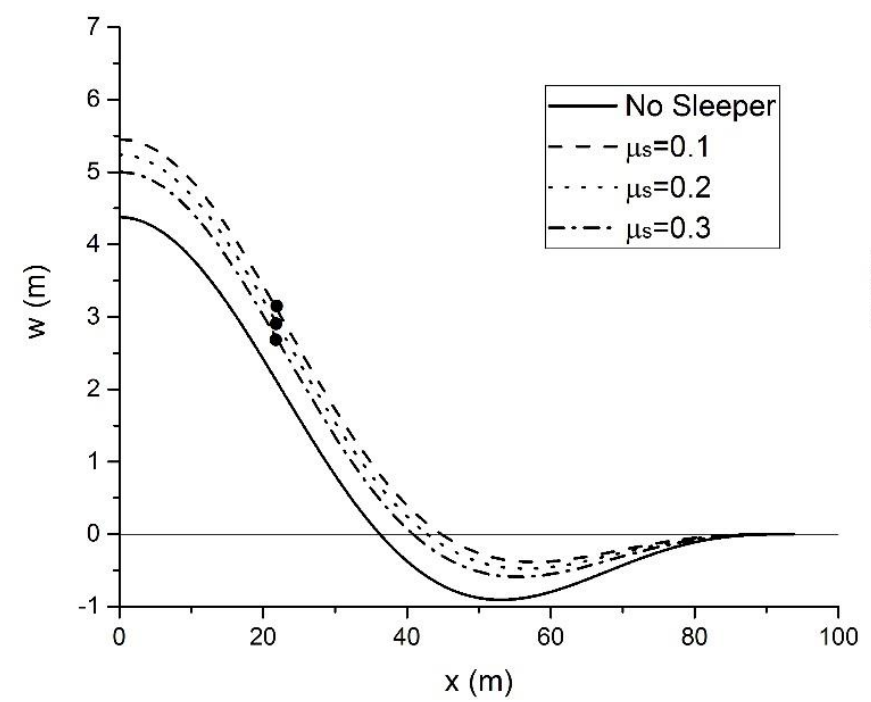

(a)

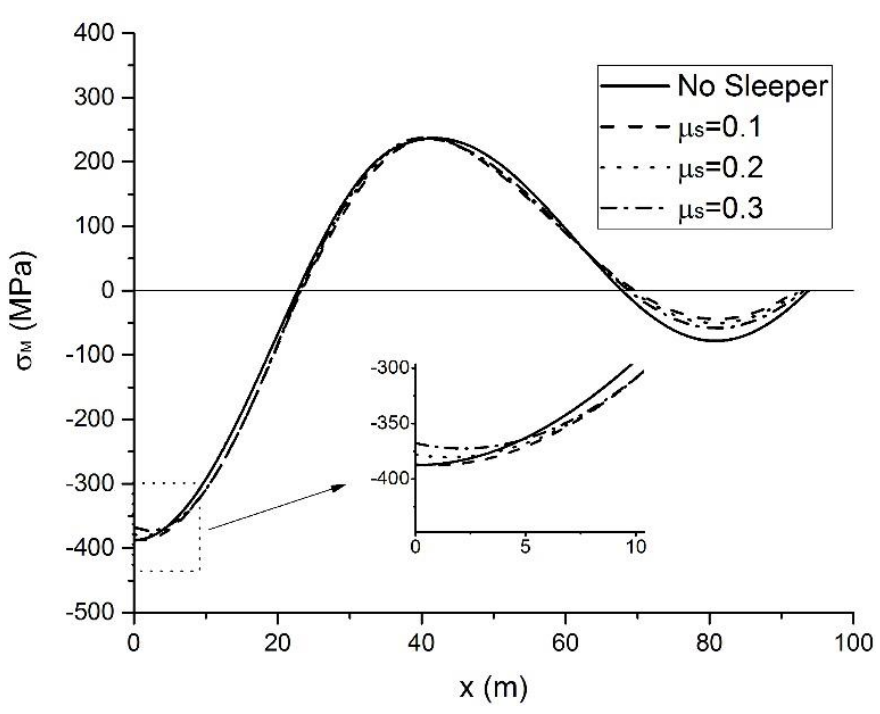

(b)

Fig. 10 Influence of sleeper friction. (a) Deformed shapes. (b) Bending stress. $v_{o m}=0.1 \mathrm{~m} . T_{0}=40{ }^{\circ} \mathrm{C}$. The dot represents the touchdown point $x=l_{1}$.

The deformed shapes and the corresponding bending stress $\sigma_{M}$ along the buckled pipeline with different values of sleeper friction under the same operating temperature difference are presented in Fig. 10-a and Fig. 10-b, respectively. From Fig. 10a we note that the primary lobe for the pipeline with sleeper is larger than that for the pipeline without sleeper and increases further with decreasing sleeper friction. However, the secondary lobe for the pipeline with sleeper is smaller than that for the pipeline without sleeper and decreases further with decreasing sleeper friction. In Fig. 10-b we see that the sleeper friction has very little influence on the bending stress. The enlarged view in Fig. 10-b shows that the bending stress at $x=0 \mathrm{~m}$ decreases with increasing sleeper friction. 


\subsubsection{Typical buckling behaviour}

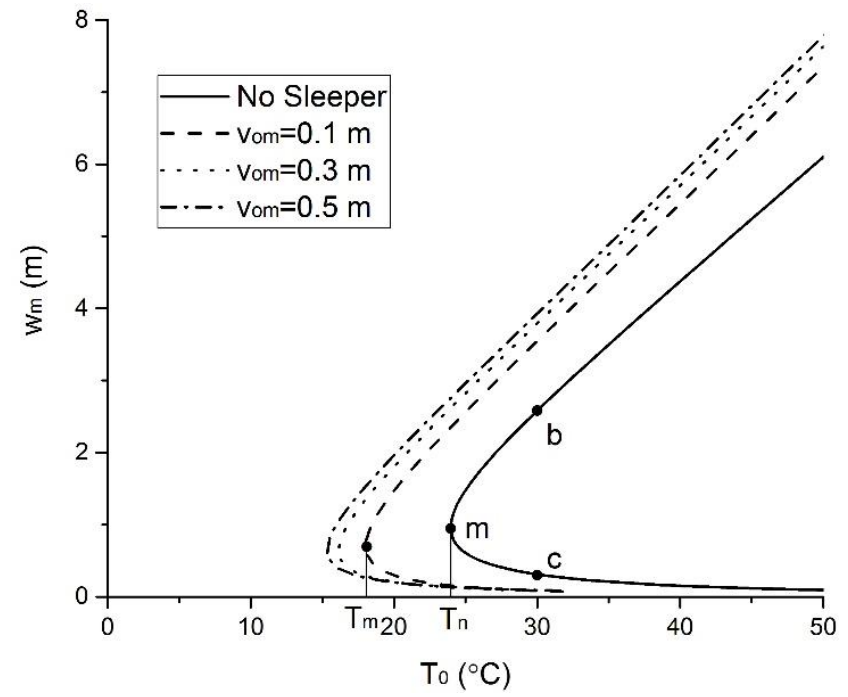

(a)

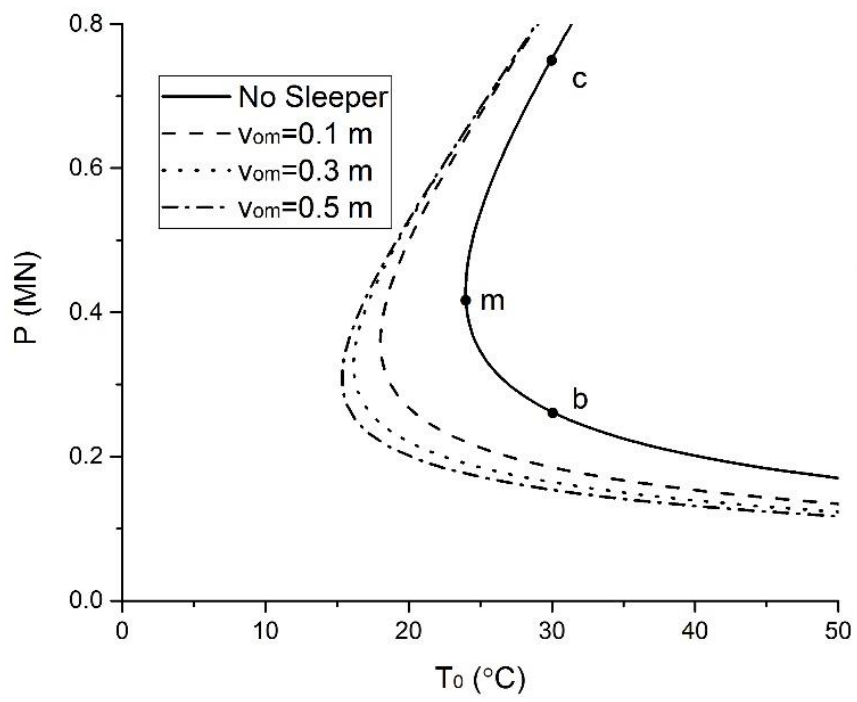

(c)

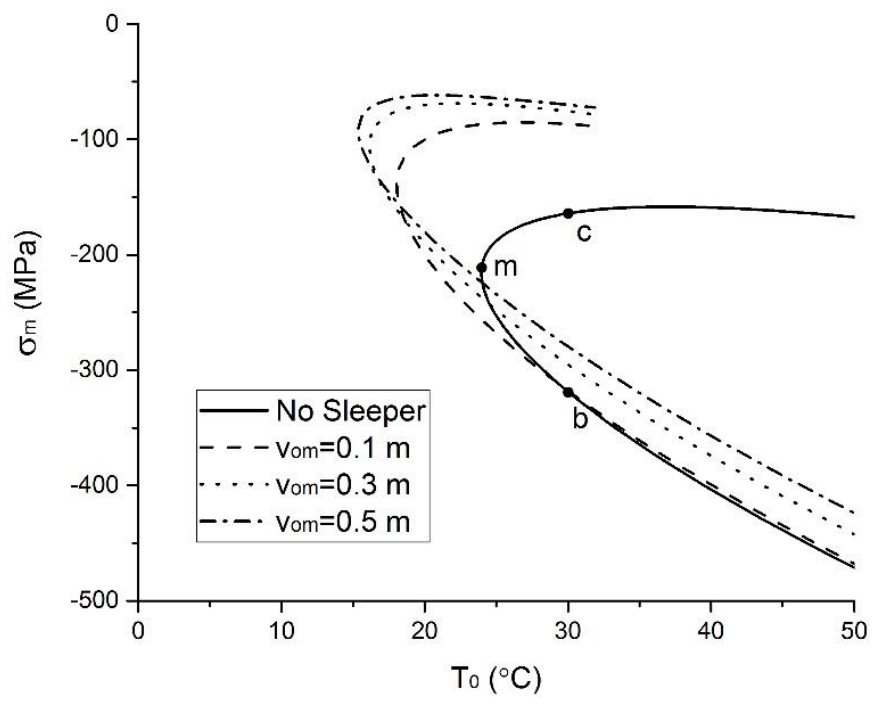

(b)

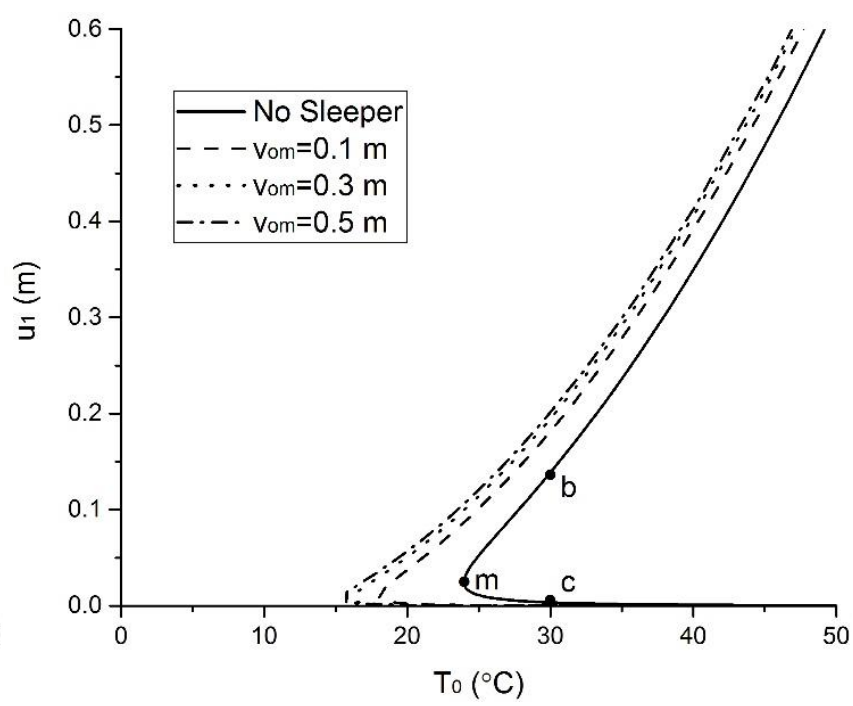

(d)

Fig. 11 Influence of sleeper height $v_{o m}$. (a) Lateral displacement amplitude $w_{m}$. (b) Maximum stress $\sigma_{m}$. (c) Axial compressive force $P$. (d) Axial thermal expansion $u_{1} \cdot \mu_{s}=0.1$.

The influence of sleeper height $v_{\text {om }}$ on typical lateral buckling behaviour is shown in Fig. 11. In Fig. 11-a the minimum critical temperature difference $T_{m}$ decreases with increasing sleeper height, which means that it will be easier for the pipeline to buckle laterally if the sleeper height is larger. Once the pipeline has buckled, the lateral displacement amplitude $w_{m}$ (see Fig. 11-a) and the maximum stress $\sigma_{m}$ (absolute value) (see Fig. 11-b) both increase with increasing temperature difference $T_{0}$. This is because the axial thermal expansion $u_{1}$ increases with increasing temperature difference (see Fig. 11-d), which means that more $u_{1}$ will feed into the buckled region, leading to a more significant lateral buckle. The lateral displacement amplitude $w_{m}$ increases and the maximum stress $\sigma_{m}$ decreases with increasing sleeper height $v_{o m}$ under the same temperature difference $T_{0}$. Moreover, the axial compressive force $P$ decreases (see Fig. 11-c) and the axial thermal expansion $u_{1}$ increases (see Fig. 11-d) with increasing temperature difference $T_{0}$. Under the same temperature difference $T_{0}$, the axial compressive force $P$ decreases and the axial thermal expansion $u_{1}$ increases with increasing sleeper height. Thus, the pipeline will be more stable for larger sleeper heights $v_{o m}$ because of a smaller axial compressive force $P$ in the post-buckling stage, if only lateral buckling is considered. However, the free span length $\left(2 l_{1}\right)$ will increase with increasing 
sleeper height and since a pipeline with larger span length has lower natural frequency, which will be closer to the fluid vortex frequency, this will more easily lead to vortex-induced vibration [42]. So both lateral buckling and vortex-induced vibration should be considered for the selection of an appropriate sleeper height $v_{o m}$.

The influence of sleeper friction $\mu_{s}$ on typical lateral buckling behaviour is shown in Fig. 12. In Fig. 12-a the minimum critical temperature difference $T_{m}$ increases with increasing sleeper friction. The lateral displacement amplitude $w_{m}$ increases (see Fig. 12-a) and the maximum stress $\sigma_{m}$ decreases (see Fig. 12-b) with decreasing sleeper friction under the same temperature difference $T_{0}$. This is because larger axial thermal expansion $u_{1}$ feeds into the buckle (see Fig. 12-d) for smaller sleeper friction, inducing larger $w_{m}$. Moreover, the primary lobe enlarges and the secondary lobe shrinks with decreasing sleeper friction under the same temperature difference (see Fig. 10-a), which makes the lateral displacement amplitude larger for smaller sleeper friction. From Fig. 12-c we note that the axial compressive force $P$ decreases with decreasing sleeper friction under the same temperature difference.

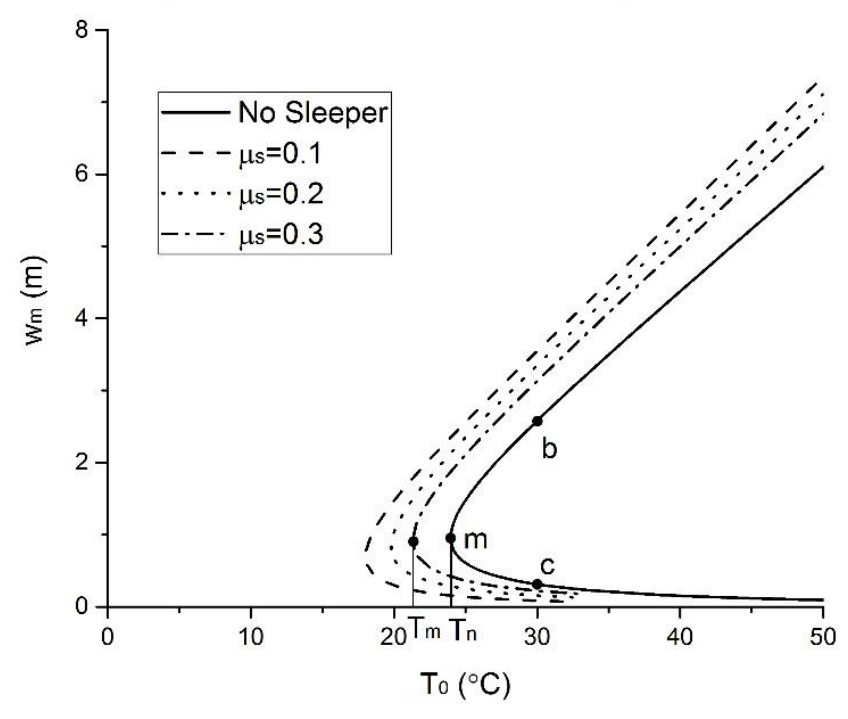

(a)

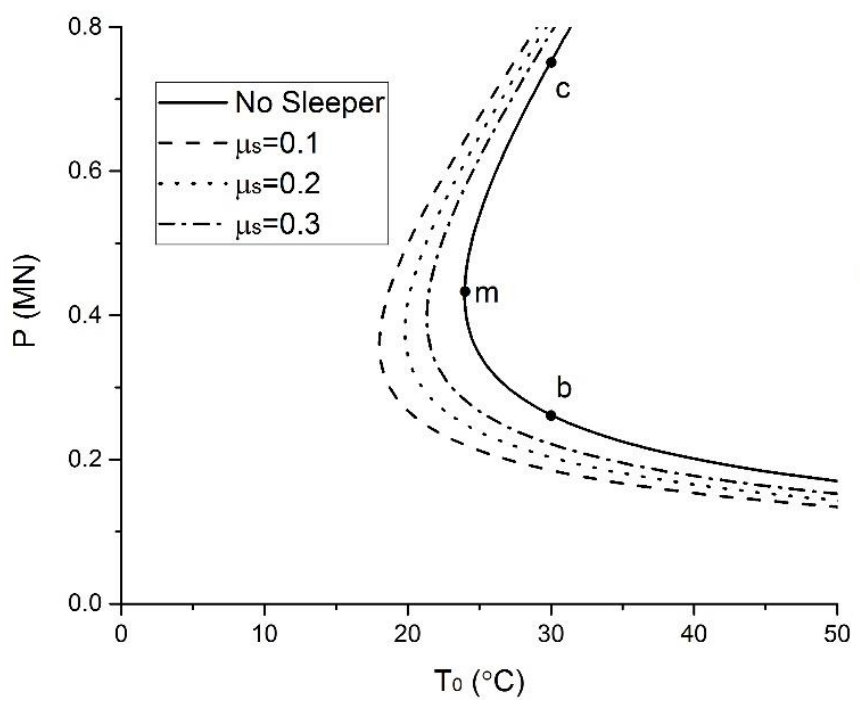

(c)

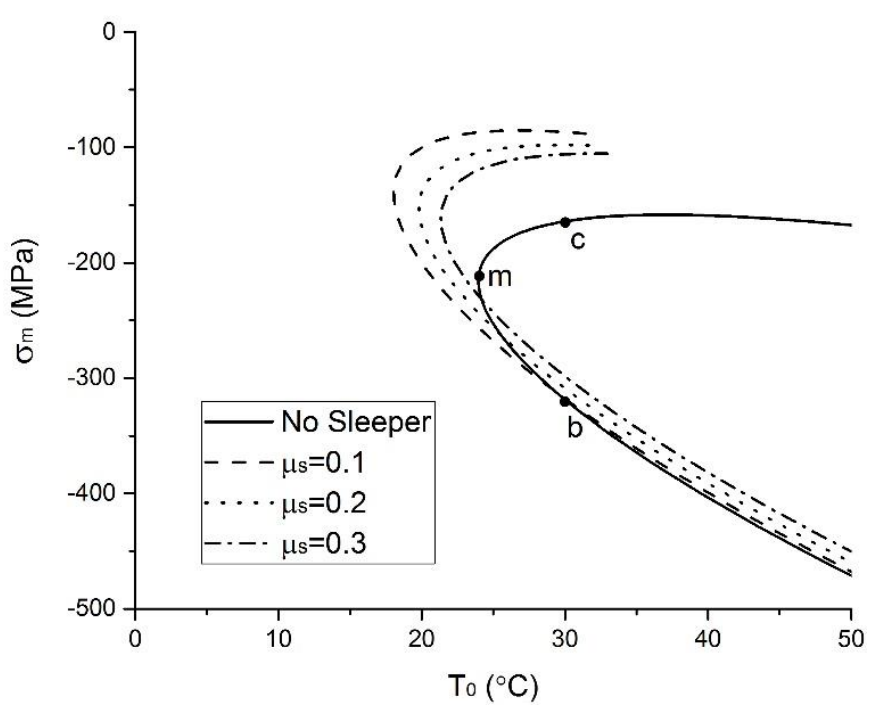

(b)

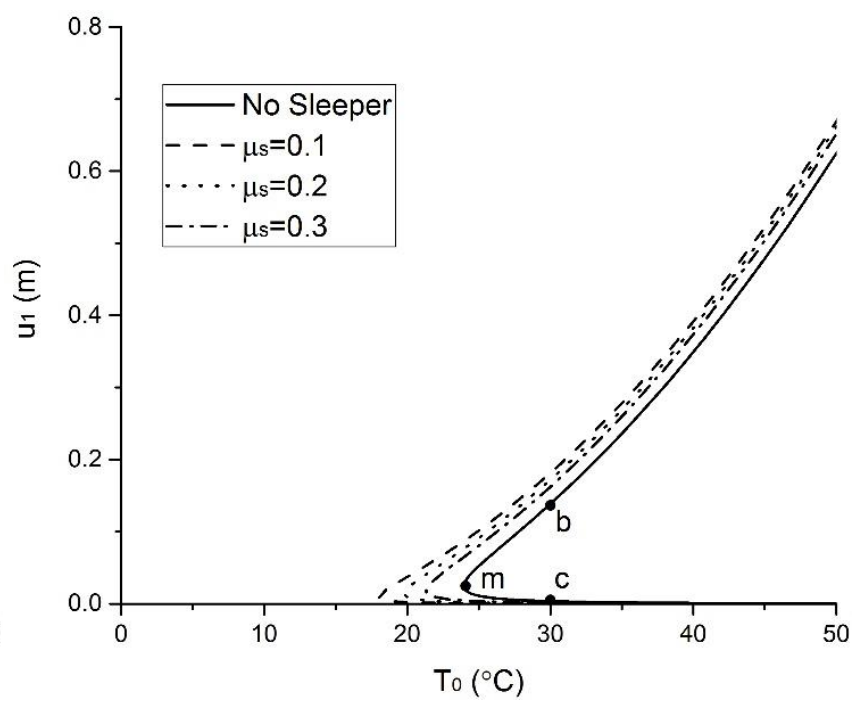

(d)

Fig. 12 Influence of sleeper friction $\mu_{s}$. (a) Lateral displacement amplitude $w_{m}$. (b) Maximum stress $\sigma_{m}$. (c) Axial compressive force $P$. (d) Axial thermal expansion $u_{1} \cdot v_{o m}=0.1 \mathrm{~m}$. 


\subsubsection{Components of maximum stress}

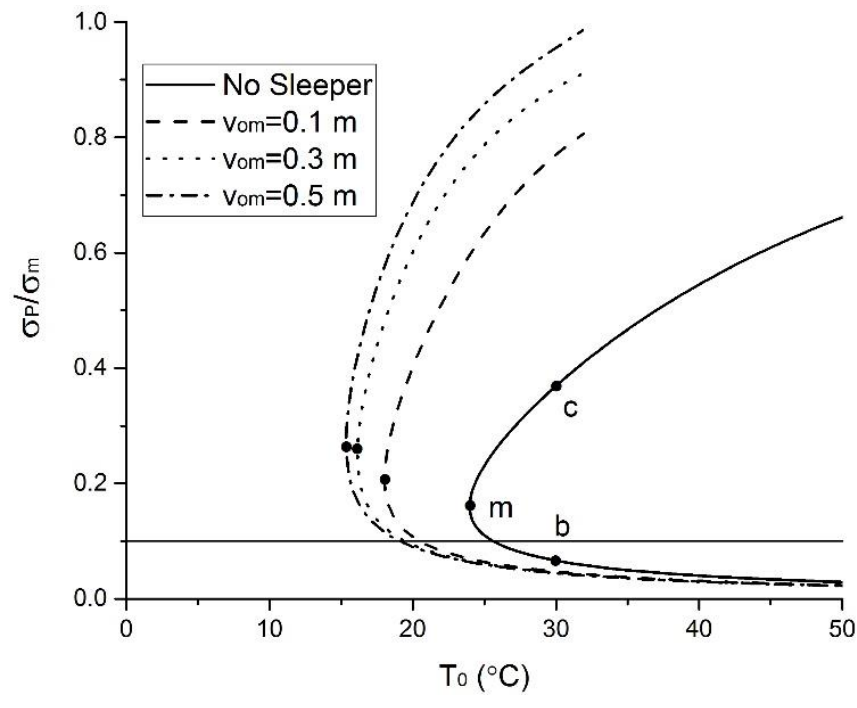

(a)

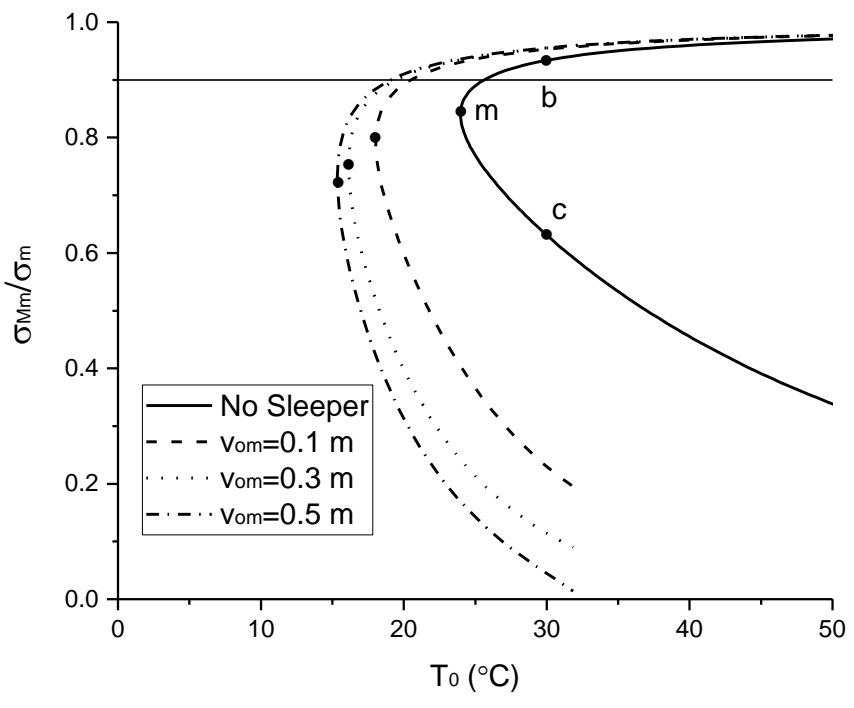

(b)

Fig. 13 Influence of sleeper height $v_{o m}$. (a) Stress ratio $\sigma_{P} / \sigma_{m}$. (b) Stress ratio $\sigma_{M m} / \sigma_{m} \cdot \mu_{s}=0.1$.

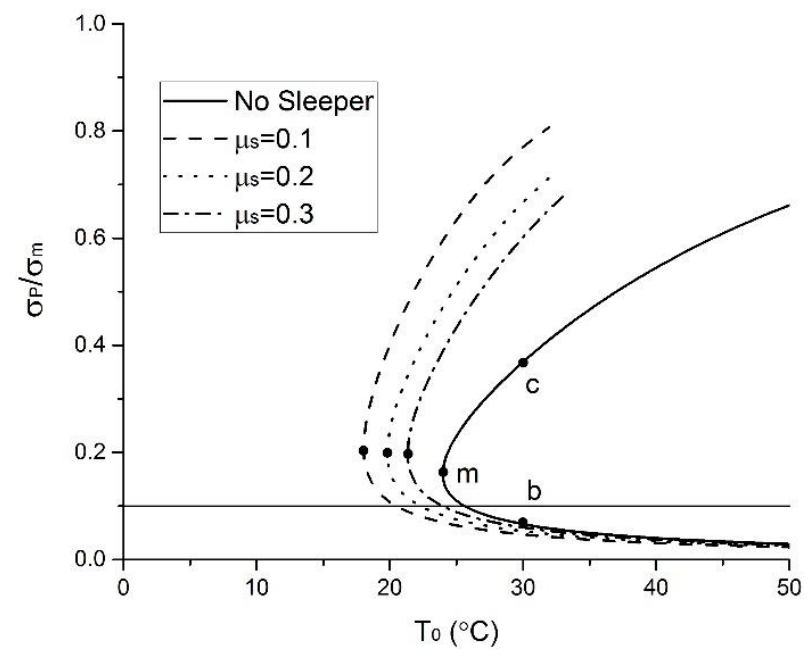

(a)

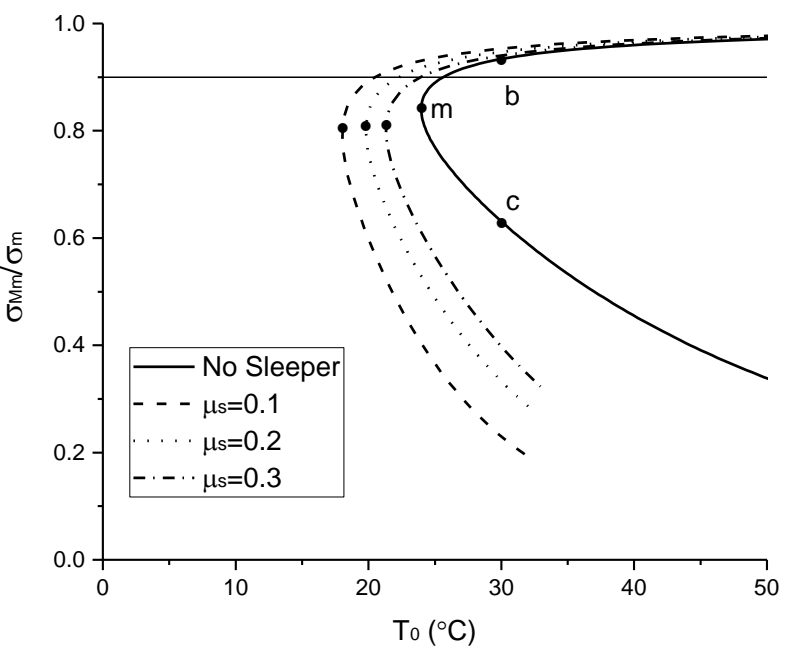

(b)

Fig. 14 Influence of sleeper friction $\mu_{s}$. (a) Stress ratio $\sigma_{P} / \sigma_{m}$. (b) Stress ratio $\sigma_{M m} / \sigma_{m} . v_{o m}=0.1 \mathrm{~m}$.

The influence of sleeper height $v_{o m}$ and sleeper friction $\mu_{s}$ on the components of the maximum stress $\sigma_{m}$ is shown in Fig. 13 and Fig. 14, respectively. The maximum stress $\sigma_{m}$ consists of two parts, namely the maximum bending stress $\sigma_{M m}$ induced by bending moment and the axial compressive stress $\sigma_{P}=P / A$ due to the post-buckling axial compressive force $P$. Branch $\mathrm{m}-\mathrm{b}$ is relatively stable while branch $\mathrm{m}-\mathrm{c}$ is relatively unstable based on the energy analysis. So only the stable branch m-b is analysed here. For each specific case, $\sigma_{P} / \sigma_{m}$ is found to decrease and $\sigma_{M m} / \sigma_{m}$ is found to increase with increasing temperature difference. In Fig. 13 and Fig. 14 it is seen that sleeper height and sleeper friction have a significant influence on the components of $\sigma_{m}$ for the unstable branch m-c. For the stable branch m-b, the influence of sleeper height and sleeper friction on the components of $\sigma_{m}$ becomes smaller with increasing temperature difference. More than $90 \%$ of the maximum stress $\sigma_{m}$ is induced by the bending moment (see Fig. 13-b and Fig. 14-b) and less than 10\% is induced by the post-buckling axial compressive force $P$ (see Fig. 13-a and Fig. 14-a) for most values of $T_{0}$. We conclude that the key point in controlling the maximum stress $\sigma_{m}$ is to control the bending stress induced by the bending moment. 


\subsubsection{Minimum critical temperature difference}

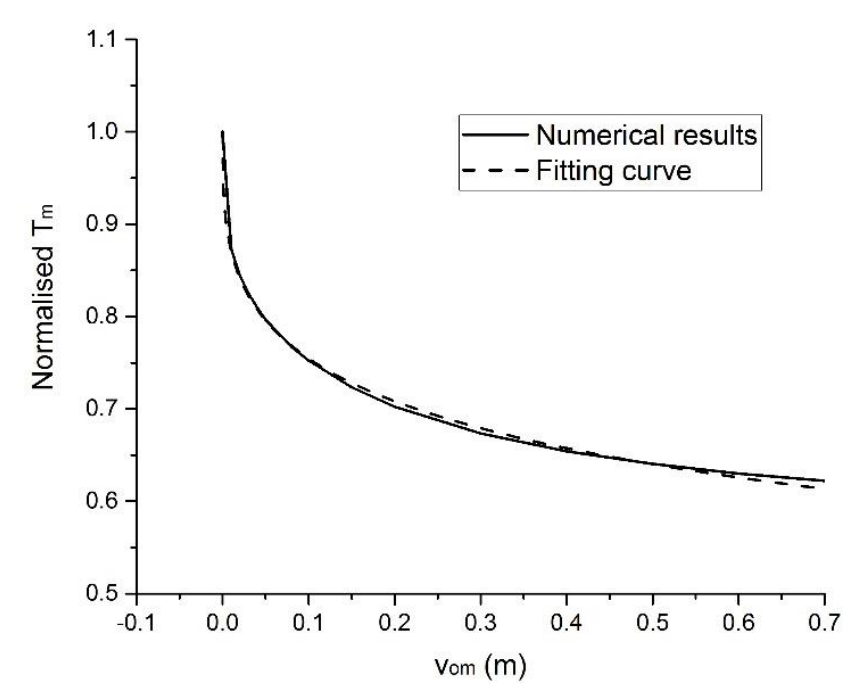

(a)

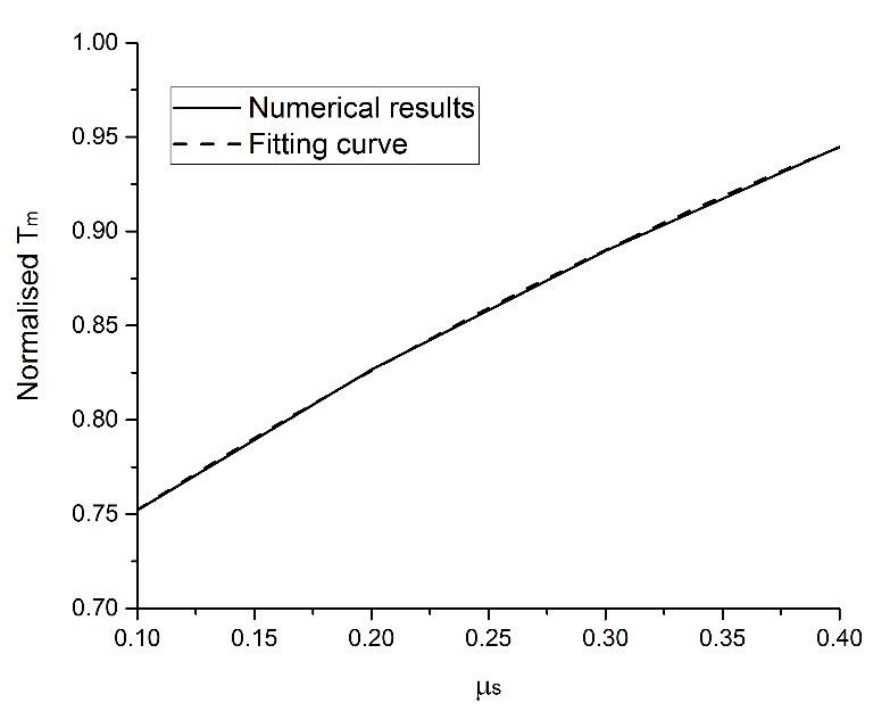

(b)

Fig. 15 Minimum critical temperature difference $T_{m}$. (a) Influence of $v_{\text {om }}$ with $\mu_{s}=0.1$. (b) Influence of $\mu_{s}$ with

$$
v_{\text {om }}=0.1 \text {. }
$$

The influence of sleeper height and sleeper friction on the minimum critical temperature difference $T_{m}$ is presented in Fig. 15-a and Fig. 15-b, respectively, in which fitting curves are also included. For pipelines without sleeper, the minimum critical temperature difference is $23.97^{\circ} \mathrm{C}$ for the data in Table 2 . The values of $T_{m}$ presented in Fig. 15 are normalised against the minimum critical temperature difference for the pipeline without sleeper. From Fig. 15-a it is obvious that there is a sharp decrease in $T_{m}$ with increasing $v_{o m}$ for small values of $v_{o m}$. Then, with further increasing $v_{o m}$, the rate of descent of $T_{m}$ reduces. For example, when $v_{o m}$ increases from $0 \mathrm{~m}$ to $0.05 \mathrm{~m}$, the normalised $T_{m}$ declines by $20 \%$. When $v_{\text {om }}$ increases from $0.05 \mathrm{~m}$ to $0.7 \mathrm{~m}$, the normalised $T_{m}$ declines by another $18 \%$. Thus the influence of $v_{o m}$ on $T_{m}$ becomes smaller with increasing $v_{o m}$. However, the possibility of inducing vortex-induced vibrations increases with increasing $v_{o m}$. So the selection of the values of $v_{o m}$ should be better evaluated. From Fig. 15-b, the normalised $T_{m}$ increases approximately linearly with increasing $\mu_{s}$. So $\mu_{s}$ should be better controlled to ensure the pipeline buckles laterally at a lower temperature difference. The numerical results in Fig. 15-a are fitted to the curve $y=1 /\left(1+d_{1} x^{d_{2}}\right)$, with, for the present data, $d_{1}=$ 0.71149 and $d_{2}=0.33973$, while the numerical results in Fig. 15-b are fitted to the quadratic polynomial $y=d_{3} x^{2}+$ $d_{4} x+d_{5}$, with $d_{3}=-0.48147, d_{4}=0.88174$ and $d_{5}=0.66893$. The fitting parameters $d_{i}(i=1-5)$ of course depend on the parameters of the pipeline and the sleeper; further parametric studies should be carried out to get universal fitting parameters.

\subsubsection{Influence of sleeper on $w_{m}$ and $\sigma_{m}$}

The influence of $v_{o m}$ on the lateral displacement amplitude $w_{m}$ and the maximum stress $\sigma_{m}$ for $T_{0}=30{ }^{\circ} \mathrm{C}$ with different sleeper friction $\mu_{s}$ is illustrated in Fig. 16, while the influence of $\mu_{s}$ on $w_{m}$ and $\sigma_{m}$ for $T_{0}=30{ }^{\circ} \mathrm{C}$ with different sleeper height $v_{o m}$ is illustrated in Fig. 17. The values of $w_{m}$ and $\sigma_{m}$ presented in Fig. 16 and Fig. 17 are normalised against the corresponding values of $w_{m}$ and $\sigma_{m}$ for the pipeline without sleeper. In Fig. 16-a it is seen that $w_{m}$ increases rapidly with increasing $v_{o m}$ for small values of $v_{o m}$. Then the rate of increase of $w_{m}$ reduces with further increase of $v_{\text {om }}$. For the same $v_{\text {om }}$, the rate of increase of $w_{m}$ is larger for smaller $\mu_{s}$. Fig. 16-b shows a sharp increase in $\sigma_{m}$ with increasing $v_{o m}$ around $0 \mathrm{~m}<v_{o m}<0.005 \mathrm{~m}$. Then, with further increasing $v_{o m}, \sigma_{m}$ decreases gradually, the rate of decrease of $\sigma_{m}$ being higher for larger $\mu_{s}$. As for the influence of $\mu_{s}$, both $w_{m}$ and $\sigma_{m}$ decrease with increasing $\mu_{s}$, as shown in Fig. 17, the rate of decrease of $w_{m}$ and $\sigma_{m}$ being higher for larger $v_{o m}$. 


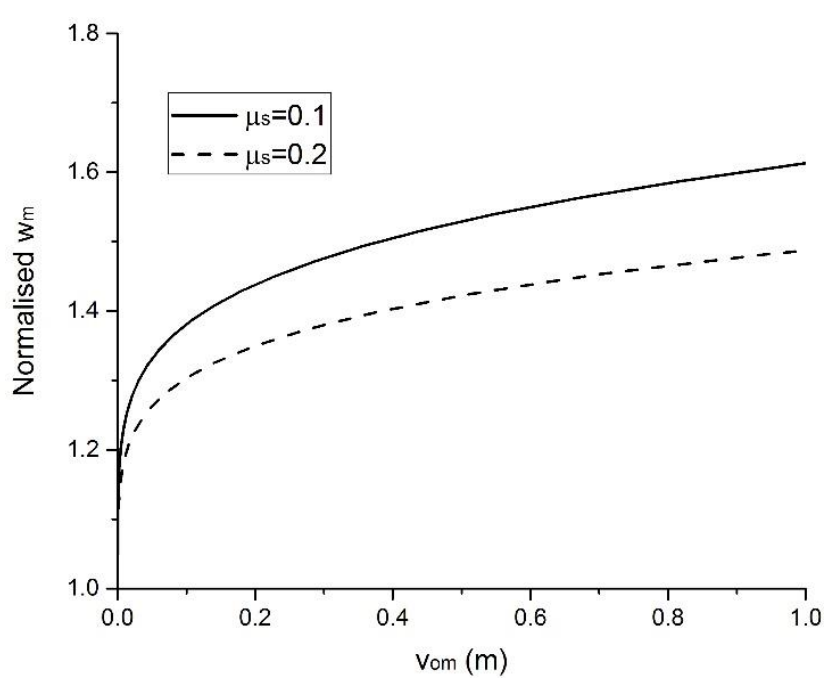

(a)

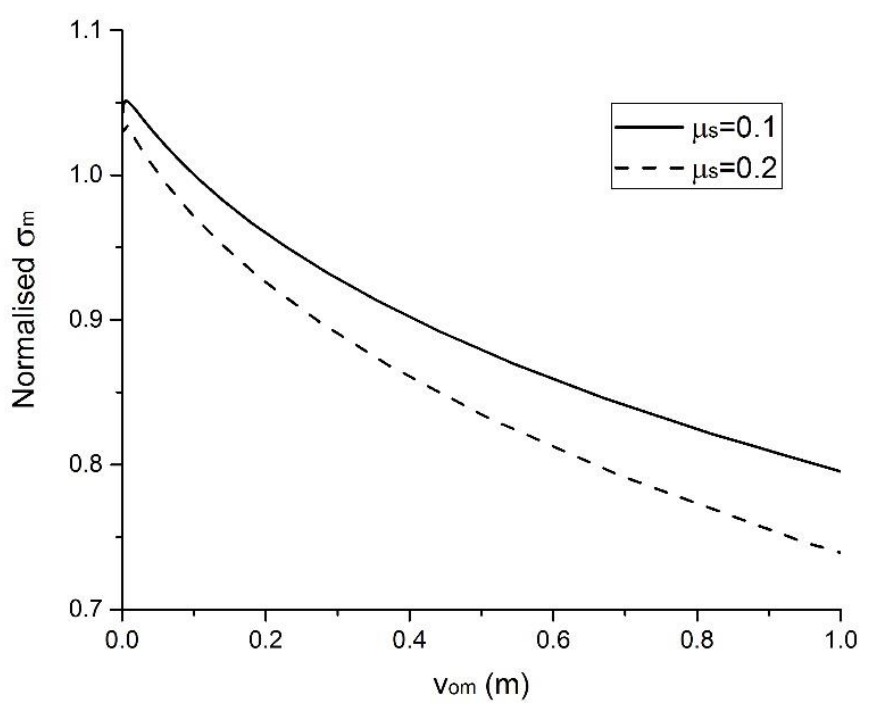

(b)

Fig. 16 Influence of sleeper height $v_{o m}$. (a) Normalised $w_{m}$. (b) Normalised $\sigma_{m} . T_{0}=30^{\circ} \mathrm{C}$.

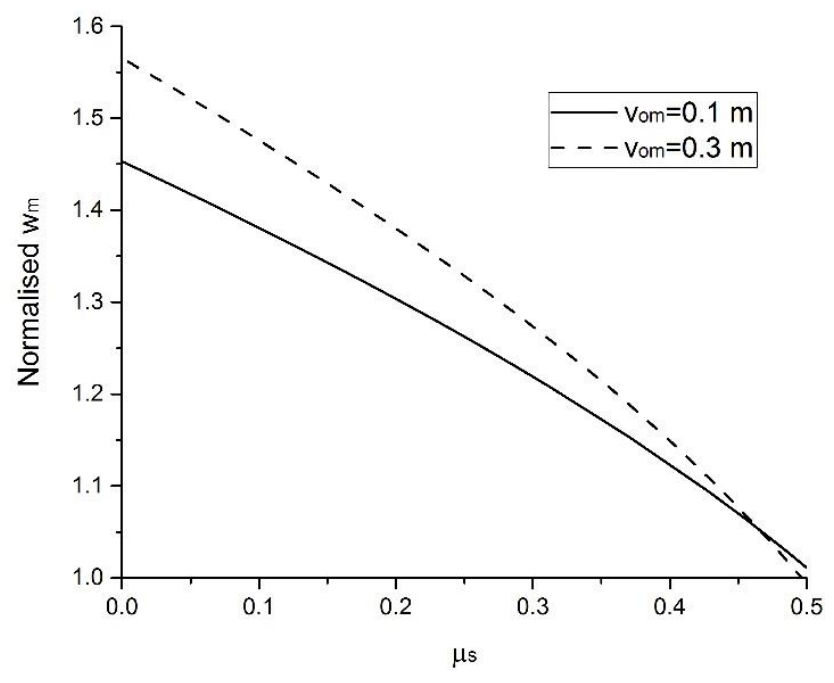

(a)

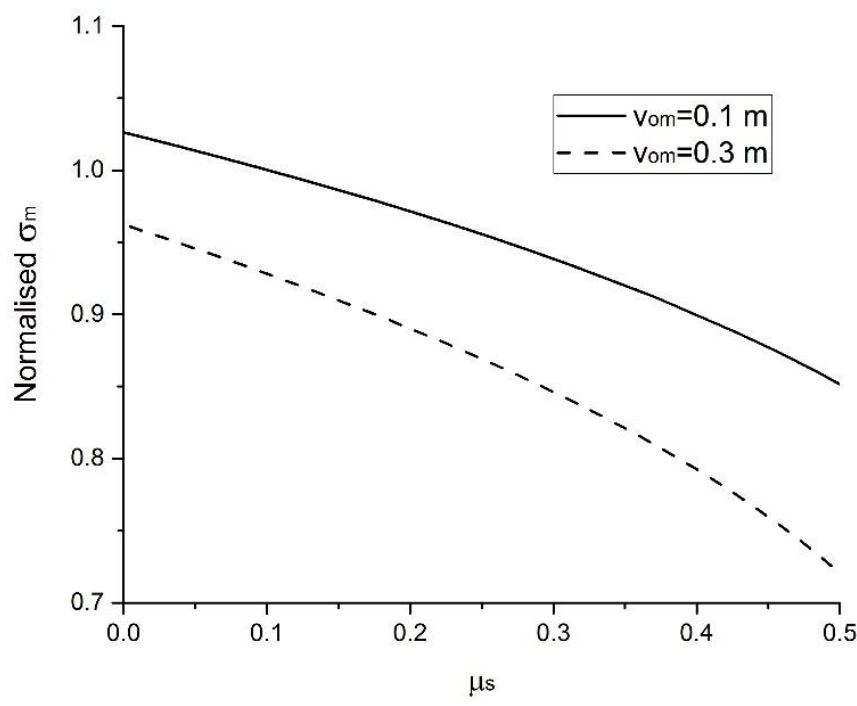

(b)

Fig. 17 Influence of sleeper friction $\mu_{s}$. (a) Normalised $w_{m}$. (b) Normalised $\sigma_{m} . T_{0}=30^{\circ} \mathrm{C}$.

\section{Conclusions}

We have derived analytical solutions for the lateral buckling of unburied subsea pipelines with a single sleeper. The solutions are third-mode solutions consisting of a primary lobe and two adjoining secondary lobes in the opposite direction. The analysis is based on small-deflection (beam) theory, but takes exact account of the compatibility between axial and lateral deformation to obtain curves of lateral deflection and stress against temperature difference $T_{0}$. We have also indicated how the model can be extended to multiple sleepers. We have validated our analytical model by comparing its predictions with experimental data, finding very good agreement. An energy analysis has been carried out to verify the stability of the two branches of lateral buckling solutions.

From our parameter studies the following conclusions can be drawn:

(i) The energy analysis reveals that one branch of lateral buckling solutions is stable and the other is unstable, as expected. Moreover, the post-buckling state is more stable for pipelines with sleeper than for pipelines without sleeper. From the comparison of the energy between the first and the third mode we conclude that third-mode buckling is more likely to happen than first-mode buckling for subsea pipelines either with or without sleeper. 
(ii) Under the same temperature difference, the primary lobe of the buckle shape for the pipeline with sleeper is larger than that for the pipeline without sleeper and grows with increasing sleeper height or with decreasing sleeper friction. However, the secondary lobe of the buckle shape has the opposite tendency. The sleeper height and the sleeper friction only have a big influence on the maximum bending stress, i.e., near $x=0$, and not on the stress distribution away from the centre of the pipe.

(iii) The minimum critical temperature difference $T_{m}$ decreases rapidly with increasing sleeper height for small values of sleeper height. However, the rate of descent of $T_{m}$ reduces with further increasing sleeper height. $T_{m}$ increases approximately linearly with increasing sleeper friction. The influence of sleeper height and sleeper friction on $T_{m}$ can be expressed well by simple curve fits.

(iv) There is a sharp increase in $w_{m}$ and $\sigma_{m}$ with increasing sleeper height within a very small interval of sleeper heights around $v_{o m}=0$. Away from this interval, $w_{m}$ increases and $\sigma_{m}$ decreases gradually with further increasing sleeper height. The rate of increase of $w_{m}$ is larger for smaller sleeper frictions, while the rate of decrease of $\sigma_{m}$ is larger for bigger sleeper frictions. Both $w_{m}$ and $\sigma_{m}$ decrease with increasing sleeper friction.

(v) Over $90 \%$ of the maximum stress is induced by the bending moment and less than $10 \%$ of the maximum stress is induced by the post-buckling axial compressive force in the post-buckling stage.

\section{Acknowledgments}

The authors would like to acknowledge that the work described in this paper was funded by the National Key Basic Research Program of China (2014CB046805). The project was also supported by the State Key Laboratory of Hydraulic Engineering Simulation and Safety (Tianjin University-HESS1510).

\section{Appendix A.}

$$
\begin{array}{r}
A_{1}=\frac{\sec \left(\lambda l_{3}\right)\left(f \cos \left(\lambda\left(l_{1}-l_{3}\right)\right)-2 f \cos \left(\lambda\left(l_{2}-l_{3}\right)\right)+f-f_{o w} \lambda \sin \left(\lambda l_{3}\right)+f_{t} \lambda \sin \left(\lambda\left(l_{1}-l_{3}\right)\right)\right)}{\operatorname{EI} \lambda^{4}} \\
A_{2}=\frac{f_{o w}}{\operatorname{EI} \lambda^{3}} \\
A_{3}=-\frac{f_{o w}}{\mathrm{E}\left[\lambda^{2}\right.} \\
A_{4}=\frac{2 l_{3}\left(-f l_{1}+2 f l_{2}+f_{o w}+f_{t}\right)+f l_{1}{ }^{2}-2 f l_{2}{ }^{2}-f l_{3}{ }^{2}-2 f_{t} l_{1}}{2 \mathrm{EI} \lambda^{2}}
\end{array}
$$

$$
B_{1}=\frac{-\tan \left(\lambda l_{3}\right)\left(-\mathrm{fsin}\left(\lambda l_{1}\right)+2 f \sin \left(\lambda l_{2}\right)+f_{o w} \lambda+f_{t} \lambda \cos \left(\lambda l_{1}\right)\right)-2 f \cos \left(\lambda l_{2}\right)+f \sec \left(\lambda l_{3}\right)}{\operatorname{EI} \lambda^{4}}
$$

$$
\begin{array}{r}
B_{2}=\frac{-\mathrm{f} \sin \left(\lambda l_{1}\right)+f_{o w} \lambda+f_{t} \lambda \cos \left(\lambda l_{1}\right)}{\mathrm{EI} \lambda^{4}} \\
B_{3}=-\frac{-f l_{1}+f_{o w}+f_{t}}{\mathrm{EI} \lambda^{2}} \\
B_{4}=\frac{\lambda^{2}\left(2 l_{3}\left(f_{o w}+f_{t}\right)-f\left(l_{3}\left(2 l_{1}+l_{3}\right)+2 l_{2}{ }^{2}-4 l_{2} l_{3}\right)\right)+2 f}{2 \mathrm{E} \lambda^{4}} \\
C_{1}=\frac{\mathrm{fsec}\left(\lambda l_{3}\right)-\tan \left(\lambda l_{3}\right)\left(-\mathrm{f} \sin \left(\lambda l_{1}\right)+2 f \sin \left(\lambda l_{2}\right)+f_{o w} \lambda+f_{t} \lambda \cos \left(\lambda l_{1}\right)\right)}{\operatorname{EI} \lambda^{4}} \\
C_{2}=\frac{-f \sin \left(\lambda l_{1}\right)+2 f \sin \left(\lambda l_{2}\right)+f_{o w} \lambda+f_{t} \lambda \cos \left(\lambda l_{1}\right)}{\operatorname{EI} \lambda^{4}} \\
C_{3}=-\frac{-f l_{1}+2 f l_{2}+f_{o w}+f_{t}}{\operatorname{EI} \lambda^{2}} \\
C_{4}=\frac{\lambda^{2} l_{3}\left(-f\left(2 l_{1}-4 l_{2}+l_{3}\right)+2 f_{o w}+2 f_{t}\right)-2 f}{2 \mathrm{EI} \lambda^{4}}
\end{array}
$$




\section{References}

[1] DNV-RP-F110. Global buckling of submarine pipelines structural design due to high temperature/high pressure. Det Norske Veritas; 2017.

[2] Bruton D, White DJ, Cheuk CY, Bolton MD, Carr M. Pipe-soil interaction behavior during lateral buckling, including largeamplitude cyclic displacement tests by the SAFEBUCK JIP (OTC-17944-MS). Offshore Technology Conference Houston, Texas 2006. p. 1-10.

[3] Dingle HRC, White DJ, Gaudin C. Mechanisms of pipe embedment and lateral breakout on soft clay. Can Geotech J. 2008;45:636-52.

[4] Wang Z, Tang Y, Zhou L, Zhao Z, Wang C. Analytical solution for controlled lateral buckling of unburied subsea pipelines. Ocean Eng. 2017;146:140-50.

[5] Shi R, Wang L. Single buoyancy load to trigger lateral buckles in pipelines on a soft seabed. Journal of Engineering Mechanics. 2015;141:1-7.

[6] Reddy R. Lateral buckling behaviour of snake-lay pipeline with vertical support at crown (OMAE2013-10106). International Conference on Ocean, Offshore and Arctic Engineering. Nantes, France 2013.

[7] Sinclair F, Carr M, Bruton D, Farrant T. Design challenges and experience with controlled lateral buckle initiation methods (OMAE2009-79434). International Conference on Ocean, Offshore and Arctic Engineering. Honolulu, Hawaii, USA 2009.

[8] Urthaler Y, Watson R, Davis J. Lateral buckling of deepwater pipelines in operation (OMAE2012-83949). International Conference on Ocean, Offshore and Arctic Engineering. Rio de Janeiro, Brazil 2012.

[9] Hobbs RE. In-service buckling of heated pipelines. Journal of Transportation Engineering. 1984;110:175-89.

[10] Taylor N, Gan AB. Submarine pipeline buckling-imperfection studies. Thin-Walled Structures. 1986;4:295-323.

[11] Ju GT, Kyriakides S. Thermal buckling of offshore pipelines. Journal of Offshore Mechanics and Arctic Engineering. 1988;110:355-64.

[12] Taylor N, Tran V. Prop-imperfection subsea pipeline buckling. Marine Structures. 1993;6:325-58.

[13] Karampour H, Albermani F, Gross J. On lateral and upheaval buckling of subsea pipelines. Engineering Structures. 2013;52:317-30.

[14] Liu R, Liu W, Wu X, Yan S. Global lateral buckling analysis of idealized subsea pipelines. Journal of Central South University. 2014;21:416-27.

[15] Hong Z, Liu R, Liu W, Yan S. Study on lateral buckling characteristics of a submarine pipeline with a single arch symmetric initial imperfection. Ocean Eng. 2015;108:21-32.

[16] Croll JGA. A simplified model of upheaval thermal buckling of subsea pipelines. Thin-Walled Structures. 1997;29:59-78.

[17] Wang L, Shi R, Yuan F, Guo Z, Yu L. Global buckling of pipelines in the vertical plane with a soft seabed. Applied Ocean Research. 2011;33:130-6.

[18] Shi R, Wang L, Guo Z, Yuan F. Upheaval buckling of a pipeline with prop imperfection on a plastic soft seabed. Thin-Walled Structures. 2013;65:1-6.

[19] Zeng X, Duan M. Mode localization in lateral buckling of partially embedded submarine pipelines. International Journal of Solids and Structures. 2014;51:1991-9.

[20] Zhu J, Attard MM, Kellermann DC. In-plane nonlinear localised lateral buckling of straight pipelines. Engineering Structures. 2015;103:37-52.

[21] Wang Z, van der Heijden GHM. Localised lateral buckling of partially embedded subsea pipelines with nonlinear soil resistance. Thin-Walled Structures. 2017;120:408-20.

[22] Maltby TC, Calladine CR. An investigation into upheaval buckling of buried pipelines-I. Experimental apparatus and some observations. International Journal of Mechanical Sciences. 1995;37:943-63.

[23] Maltby TC, Calladine CR. An investigation into upheaval buckling of buried pipelines-II. Theory and analysis of 
experimental observations. International Journal of Mechanical Sciences. 1995;37:965-83.

[24] Karampour H, Albermani F. Experimental and numerical investigations of buckle interaction in subsea pipelines. Engineering Structures. 2014;66:81-8.

[25] Karampour H, Albermani F, Veidt M. Buckle interaction in deep subsea pipelines. Thin-Walled Structures. 2013;72:113-20.

[26] Wang Z, Chen Z, Liu H, Bu Y. Static and dynamic analysis on upheaval buckling of unburied subsea pipelines. Ocean Eng. 2015;104:249-56.

[27] Wang Z, Chen Z, Liu H. Numerical study on upheaval buckling of pipe-in-pipe systems with full contact imperfections. Engineering Structures. 2015;99:264-71.

[28] Liu R, Xiong H, Wu X, Yan S. Numerical studies on global buckling of subsea pipelines. Ocean Eng. 2014;78:62-72.

[29] Liu R, Basu P, Xiong H. Laboratory tests and thermal buckling analysis for pipes buried in Bohai soft clay. Marine Structures. 2015;43:44-60.

[30] Hong Z, Liu R, Liu W, Yan S. A lateral global buckling failure envelope for a high temperature and high pressure (HT/HP) submarine pipeline. Applied Ocean Research. 2015;51:117-28.

[31] Zhang X, Duan M. Prediction of the upheaval buckling critical force for imperfect submarine pipelines. Ocean Eng. 2015;109:330-43.

[32] Zeng X, Duan M, Che X. Critical upheaval buckling forces of imperfect pipelines. Applied Ocean Research. 2014;45:33-9.

[33] Peek R, Yun H. Flotation to trigger lateral buckles in pipelines on a flat seabed. Journal of Engineering Mechanics. 2007;4:442-51.

[34] Wang Z, Tang Y, Wang C. Analytical solution for lateral buckling of unburied subsea pipelines with distributed buoyancy section. Ocean Eng. 2017;146:115-24.

[35] Li G, Zhan L, Li H. An analytical solution to lateral buckling control of subsea pipelines by distributed buoyancy sections. Thin-Walled Structures. 2016;107:221-30.

[36] Wang Z, Tang Y, van der Heijden GHM. Analytical study of distributed buoyancy sections to control lateral thermal buckling of subsea pipelines. Marine Structures. 2018;58:199-222.

[37] Silva-Junior HC, Cardoso CO, Carmignotto MAP, Zanutto JC. Reduced Model Device of Solutions to Control Thermal Buckling Effects in HP-HT Subsea Pipelines (OMAE2008-57637). International Conference on Ocean, Offshore and Arctic Engineering. Estoril, Portugal 2009.

[38] de Oliveira Cardoso C, Solano RF. Performed of triggers to control thermal buckling of subsea pipelines using reduced scale model (ISOPE-I-15-445). International Offshore and Polar Engineering Conference. Hawaii, USA 2015.

[39] Wang Z, Tang Y, van der Heijden GHM. Analytical study of lateral thermal buckling for subsea pipelines with sleeper. ThinWalled Structures. 2018;122:17-29.

[40] Bai Q, Qi X, Brunner M. Global buckle control with dual sleepers in HP/HT pipelines (OTC-19888-MS). Offshore Technology Conference. Texas, USA 2009.

[41] Wang Z, Chen Z, Liu H, Zhang Z. Numerical study on lateral buckling of pipelines with imperfection and sleeper. Applied Ocean Research. 2017;68:103-13.

[42] DNV-RP-F105. FREE SPANNING PIPELINES. 2006. 